\title{
High-resolution CO observation of the carbon star CIT 6 revealing the spiral structure and a nascent bipolar outflow
}

\author{
Hyosun $\operatorname{Kim}^{1,2,3}$ \\ Sheng-Yuan Liu ${ }^{1}$ \\ Naomi Hirano ${ }^{1}$ \\ Ronny Zhao-Geisler ${ }^{4,1}$ \\ Alfonso Trejo ${ }^{1}$ \\ Hsi-Wei Yen ${ }^{1}$ \\ Ronald E. Taam ${ }^{1}$ \\ Francisca Kemper ${ }^{1}$ \\ Jongsoo Kim ${ }^{2}$ \\ Do-Young Byun ${ }^{2}$ \\ Tie $\mathrm{Liu}^{2}$
}

\begin{abstract}
CIT 6 is a carbon star in the transitional phase from the asymptotic giant branch (AGB) to the protoplanetary nebulae $(\mathrm{pPN})$. Observational evidences of two point sources in the optical, circumstellar arc segments in an $\mathrm{HC}_{3} \mathrm{~N}$ line emission, and a bipolar nebula in near-infrared provide strong support for the presence of a binary companion. Hence, CIT 6 is very attractive for studying the role of companions in the AGB-pPN transition. We have carried out high resolution ${ }^{12} \mathrm{CO} J=2-1$ and ${ }^{13} \mathrm{CO}$ $J=2-1$ observations of CIT 6 with the Submillimeter Array combined with the Submillimeter Telescope (single-dish) data. The ${ }^{12} \mathrm{CO}$ channel maps reveal a spiral-shell
\end{abstract}

\footnotetext{
${ }^{1}$ Academia Sinica Institute of Astronomy and Astrophysics, P.O. Box 23-141, Taipei 10617, Taiwan; hkim@asiaa.sinica.edu.tw

${ }^{2}$ Korea Astronomy and Space Science Institute, 776, Daedeokdae-ro, Yuseong-gu, Daejeon 305-348, Korea

${ }^{3}$ EACOA fellow

${ }^{4}$ National Taiwan Normal University, Department of Earth Sciences, 88 Sec. 4, Ting-Chou Rd, Wenshan District, Taipei 11677, Taiwan
} 
pattern connecting the $\mathrm{HC}_{3} \mathrm{~N}$ segments in a continuous form, and an asymmetric outflow corresponding to the near-infrared bipolar nebula. Rotation of the ${ }^{12} \mathrm{CO}$ channel peak position may be related to the inner spiral winding and/or the bipolar outflow. An eccentric orbit binary is suggested for the presences of an anisotropic mass loss to the west and a double spiral pattern. The lack of interarm emission to the west may indicate a feature corresponding to the periastron passage of a highly eccentric orbit of the binary. Spatially-averaged radial and spectral profiles of ${ }^{12} \mathrm{CO} J=2-1$ and ${ }^{13} \mathrm{CO} J=2-1$ are compared with simple spherical radiative transfer models, suggesting a change of ${ }^{12} \mathrm{CO} /{ }^{13} \mathrm{CO}$ abundance ratio from $\sim 30$ to $\sim 50$ inward in the CSE of CIT 6 . The millimeter continuum emission is decomposed into extended dust thermal emission (spectral index $\sim-2.4$ ) and compact emission from radio photosphere (spectral index $\sim-2.0)$.

Subject headings: circumstellar matter — stars: AGB and post-AGB — stars: individual (CIT 6) — stars: late-type — stars: mass-loss — stars: winds, outflows

\section{INTRODUCTION}

Beyond the main-sequence of a stellar population, a star with an initial mass less than $8 M_{\odot}$ evolves to the red giant phase at which it ejects most of its mass through a slow and dense wind $\left(3-30 \mathrm{~km} \mathrm{~s}^{-1}\right)$. The intense mass loss process reaches its climax at the tip of the asymptotic giant branch (AGB) with a rate of $10^{-7}-10^{-4} M_{\odot} \mathrm{yr}^{-1}$ forming an extensive circumstellar envelope (CSE, $\left.10^{16}-10^{18} \mathrm{~cm}\right)$. Such an evolutionary phase leads to the obscuration of the innermost envelope region near the stellar surface. Consequently, information on the onset of dust formation and growth, the subsequent wind acceleration, the central engine governing the (a)symmetric shapes of the entire CSE, and the connection of these features with binary interactions and/or magnetic fields is very limited.

The morphological diversity of the CSEs in evolved stellar phases is commonly assumed to be closely related to the possible binary nature of the system. In contrast to quasi-spherical shapes of CSEs at the AGB, the majority of planetary nebulae (PNe) or protoplanetary nebulae (pPNe) bear bipolar or multipolar structures, often observed in ionized gas or dust scattered light (e.g., Corradi \& Schwarz 1995; Balick \& Frank 2002; Sahai et al. 2007). Such bipolarity is a common feature of sources possessing accretion disks such as the protoplanetary jets coexisting with the accretion disks surrounding young stellar objects. In the outflowing CSE of evolved giant stars, another gravitational potential (e.g., companion) is hypothesized to assemble material in the form of circumstellar or circumbinary disk to feed the observed bipolar outflow. The accretion disks may form during the AGB phase in close binary systems (e.g., Theuns \& Jorissen 1993; Huarte-Espinosa et al. 2013).

Wide binary systems also induce, albeit less vigorous, asymmetry in the form of spiral pat- 
terns. It is now theoretically well understood that binary orbital motion leads to a spiral-shell pattern propagating over the entire CSE (e.g., Soker 1994; Mastrodemos \& Morris 1999; Mohamed \& Podsiadlowski 2012; Kim \& Taam 2012a b). The consequent wind anisotropy, caused by the binary orbital motion and the gravitational focusing of the wind material onto the companion, leads an overall flattened geometry of the CSE (Huggins et al. 2009; Kim \& Taam 2012b). The binary-induced pattern and geometry of CSE preserve the records of binary influence on the wind outflow. A well-defined spiral pattern of AFGL 3068 found from dust scattered light and the binary separation inferred from near-infrared adaptive optics images (Morris et al. 2006; Mauron \& Huggins 2006) were utilized to derive its binary stellar and orbital properties (Kim \& Taam 2012c). The non-continuous shells within the CSE of CIT 6, which appeared in a molecular line emission (Claussen et al. 2011), were suggested to be a spiral-shell pattern when viewed with a large inclination from the orbital plane (Kim et al. 2013). Such an interpretation may also be applicable to the multiple rings and arcs often found in the CSEs of AGB stars and pPNe, e.g., IRC+10216 (Mauron \& Huggins 1999; Decin et al. 2015; Cernicharo et al. 2015) and Mira (Mayer et al. 2011; Ramstedt et al. 2014). In addition, within this framework, the wind properties derived from the circumstellar spiral pattern facilitated constraints on the mass loss history of R Sculptoris (Maercker et al. 2012). Finally, the elliptical shapes of CSEs of AGB stars in an optical imaging survey were suggested as a probe to infer that a significant fraction of AGB stars may have hidden binary companions (Mauron et al. 2013).

In fact, substantial pPNe may reveal evidence of the shape transition from outer rings and arcs formed in the CSE during the previous AGB phase to the inner region showing bipolar lobes developed by new vigorous ejections (e.g., Egg nebula, Sahai et al. 1998). If the ring-like patterns originated from binary orbital motion, the typical dynamical timescales of the observed patterns (100-1000 years) correspond to the orbital periods of wide binary systems with the companions locating at a few tens to hundred AU. Such separations between binary stars are far larger than radius of wind acceleration zone so that the wind speed is sufficiently fast in the vicinity of the companion (if it is in a circular orbit), precluding formation of an accretion disk and consequently a bipolar outflow. Whether a wide binary can create a bipolar outflow has not been well studied. The onset of the asymmetric outflow, the role of a binary companion on it, and ultimately the mass loss process may be addressed by sub-arcsecond resolution observations toward the sources in this phase transition from the AGB to $\mathrm{pPN}$.

The extreme carbon star CIT 6 is an excellent target to study the role of a companion on the transition from a quasi-spherical shell-like structure (over $\sim 20^{\prime \prime}$ ) found in a molecular line emission map (Claussen et al. 2011) to a nascent bipolar structure $\left(\lesssim 2^{\prime \prime}\right)$ observed in the near-infrared polarization image (Schmidt et al. 2002). The companion candidate revealed in optical images is $\lesssim 0^{\prime \prime} .2$ away from the carbon star at a position angle (PA, from north to east) of $\sim 10^{\circ}$ Monnier et al. 2000). At a distance of $400 \mathrm{pc}$ (Cohen \& Hitchon 1996, and references therein) derived by using a period-luminosity relation from the pulsation period of the carbon star $(\sim 1.65 \mathrm{yr})$, the projected binary separation corresponds to $\lesssim 80 \mathrm{AU}$. The observed flux at $4500 \AA$ suggests the 
candidate companion to be a main-sequence star of mass $\sim 1-2 M_{\odot}($ Schmidt et al. 2002). On the other hand, the effective temperature $\sim 2800 \mathrm{~K}($ Cohen 1979$)$ and bolometric luminosity $\sim 10^{4} L_{\odot}$ (Loup et al. 1993) locate the carbon star CIT 6 at the tip of AGB of a star with an initial mass of $\sim 2 M_{\odot}$ (current mass $\left.\lesssim 1 M_{\odot}\right)$ based on the stellar evolutionary model with solar metallicity using the code provided by Hurley et al. (2000). These photometric masses agree with the masses derived from a separate, parameter space analysis based on binary dynamics and the encoded circumstellar pattern (Kim et al. 2013).

The circumstellar pattern of CIT 6 was observed in the $\mathrm{HC}_{3} \mathrm{~N} J=4-3$ line emission at a high resolution of $\sim 0$.'7 with the Karl G. Jansky Very Large Array (JVLA). The pattern was modeled with spherical shells, resulting in a necessary displacement of shell centroids along the radius and across the line velocity channels (Claussen et al. 2011; see also Chau et al. 2012 for various $\mathrm{HC}_{3} \mathrm{~N}$ and $\mathrm{HC}_{5} \mathrm{~N}$ lines of CIT 6 ). This departure from spherical symmetry is consistent with the spiralshell model induced by a binary motion, and Kim et al. (2013) indeed presented a spiral-shell model reproducing the observed $\mathrm{HC}_{3} \mathrm{~N}$ pattern in all channels. Previously, Dinh-V.-Trung \& Lim (2009) speculated a spiral structure of CIT 6 from another $\mathrm{HC}_{3} \mathrm{~N} J=5-4$ emission map at a lower resolution of $\sim 1^{\prime \prime} .5$, which is now resolved into a few windings of the modeled spiral-shell pattern. Nevertheless, the pattern geometry was left incomplete, because the observed $\mathrm{HC}_{3} \mathrm{~N}$ pattern is broken with an absence of emission to the west and in the central $\sim 3^{\prime \prime}$ region. Verifying the pattern shape with a chemically stable molecule such as ${ }^{12} \mathrm{CO}$ is necessary to infer the companion of CIT 6, to examine the binary characteristics, and to obtain a clue of the kinematic connection of the spiral pattern to the observed near-infrared bipolar nebula in the innermost region where the $\mathrm{HC}_{3} \mathrm{~N}$ emission was absent.

In this paper, we report the Submillimeter Array ${ }^{1}$ (SMA) observations toward CIT 6 in the ${ }^{12} \mathrm{CO} J=2-1$ and ${ }^{13} \mathrm{CO} J=2-1$ lines and the $226.2 \mathrm{GHz}$ and $350.6 \mathrm{GHz}$ continuum emission at sub-arcsecond resolutions. Submillimeter Telescope (SMT) grid-mapping observations for the same lines are combined with the SMA data to recover the extended CSE emission. We describe the observations and calibrations in Section 2, The results are presented in Sections 3 and 4 for the overall and fine structures in the CSE of CIT 6, respectively. Further discussion follows in Section 5. We summarize our findings in Section6, and conclude in Section7.

\footnotetext{
${ }^{1}$ The Submillimeter Array is a joint project between the Smithsonian Astrophysical Observatory and the Academia Sinica Institute of Astronomy and Astrophysics, and is funded by the Smithsonian Institution and the Academia Sinica.
} 


\section{OBSERVATIONS}

\subsection{SMA Observations}

The ${ }^{12} \mathrm{CO} J=2-1$ and ${ }^{13} \mathrm{CO} J=2-1$ lines and the adjacent continuum (at $226.2 \mathrm{GHz}$ ) have been simultaneously observed with the SMA in four different configurations (see Table1). These configurations provided the projected baselines ranging from $\sim 5 \mathrm{~m}$ to $\sim 500 \mathrm{~m}$. The observations were carried out on five different dates. On 2013 Jan. 08 and Feb. 06 for extended and very extended configurations, two receivers were simultaneously setup with one receiver at $\sim 226 \mathrm{GHz}$ covering ${ }^{12} \mathrm{CO} J=2-1$ and ${ }^{13} \mathrm{CO} J=2-1$, and the other receiver at $\sim 350 \mathrm{GHz}$. Each receiver has $2 \mathrm{GHz}$ IF-bandwidth. On 2013 Dec. 12, 2014 Feb. 09, and 2014 Mar. 11, however, one $230 \mathrm{GHz}$ receiver was setup with $4 \mathrm{GHz}$ IF-bandwidth. Several lines were detected, but were spatially unresolved; hence, in this paper, we focus on the ${ }^{12} \mathrm{CO} J=2-1$ and ${ }^{13} \mathrm{CO} J=2-1$ lines. The primary beam sizes of the SMA antenna are $\sim 55^{\prime \prime}$ at $230 \mathrm{GHz}$ and $\sim 36^{\prime \prime}$ at $350 \mathrm{GHz}$, respectively.

The visibility data were calibrated with the standard procedure using the MIR packag $\rfloor^{2}$ with two quasars $0854+201$ and $0927+390$ as the gain (phase and amplitude) calibrators, and a quasar, either 3C84 or 3C279, as the bandpass calibrator. The flux calibrations were mainly based on the SMA flux monitoring program of a large number of quasars, tabulated in the Tools section of

the SMA Observer Center (Gurwell et al. 2007); when Titan or Callisto is available, the fluxes of the observed quasars measured based on the planetary object were consistent with the monitoring records.

The MIRIAD packages were used for the inverse Fourier transformation and image cleaning from the calibrated visibility data to the final channel maps. The synthesized beam size of the ${ }^{12} \mathrm{CO}$ $J=2-1$ line emission map is $0.56 \times 0^{\prime \prime} .44$ with a PA of $40^{\circ}$ with the uniform weighting of the visibility data from all configurations listed in Table1. The velocity interval was set to be $1 \mathrm{~km} \mathrm{~s}^{-1}$ in the imaging step. The noise level $\sigma$ estimated from the line-free channels is 24 mJy beam $^{-1}(2.2 \mathrm{~K})$ at the velocity resolution of $1 \mathrm{~km} \mathrm{~s}^{-1}$. The continuum map at $226.2 \mathrm{GHz}$ was attained by averaging over the line-free channels of both sidebands (separated by $10 \mathrm{GHz}$ ) with uniform weighting of the data from all SMA configurations. Its synthesized beam has a size of $0.54 \times 00^{\prime \prime} 43$ with a PA of $40^{\circ}$, and the noise level is $0.75 \mathrm{mJy}^{\text {beam }}{ }^{-1}(76 \mathrm{mK})$. The $350.6 \mathrm{GHz}$ continuum imaging is characterized by a synthesized beam of $0^{\prime \prime} 36 \times 0^{\prime \prime} .30$ with a PA of $25^{\circ}$ and a noise of $3.6 \mathrm{mJy}^{\circ}$ beam $^{-1}(330 \mathrm{mK})$.

\subsection{SMT Observations}

The SMT grid-mapping observations have been carried out to fill in the zero and short baselines of the ${ }^{12} \mathrm{CO} J=2-1$ and ${ }^{13} \mathrm{CO} J=2-1$ lines using the $1.3 \mathrm{~mm}$ sideband-separating receivers with dual-polarization. The integration per pointing of the grid-mapping is $3 \mathrm{~min}$ for ${ }^{12} \mathrm{CO}$ and $6 \mathrm{~min}$ for

${ }^{2}$ See https: //www.cfa.harvard.edu/ cqi/mircook.html 
${ }^{13} \mathrm{CO}$ (see Table 2). Deep single-point observations toward the central position were additionally performed; the total integration for ${ }^{12} \mathrm{CO}$ central pointing is $11 \mathrm{~min}$, and $392 \mathrm{~min}$ for ${ }^{13} \mathrm{CO}$. A 1024 channel $(2 \times 512)$ backend with a $1 \mathrm{MHz}$ filterbank was employed. The On/Off-integrations were repeated to correct the sky background variation via position-switching for the grid-mapping mode and beam-switching for the single-point mode. Pointing corrections were monitored by observations of Jupiter. The beam size of a single pointing is $\sim 33^{\prime \prime}$ at $1.3 \mathrm{~mm}$.

Using the CLASS software package in GILDAS 3 , we subtracted the linear baseline fits, and co-added the individual scans to achieve the final spectra. The temperature scales, $T_{\mathrm{A}}^{*}$, of ${ }^{12} \mathrm{CO}$ and ${ }^{13} \mathrm{CO}$ lines are $2.95 \mathrm{~K}$ and $0.20 \mathrm{~K}$, respectively, at the peaks, comparable (with $<20 \%$ uncertainty) to the values in earlier SMT single-pointing observations $\left(2.49 \mathrm{~K}\right.$ for ${ }^{12} \mathrm{CO}$ and $0.22 \mathrm{~K}$ for ${ }^{13} \mathrm{CO}$, by Milam et al. 2009). The main-beam temperatures were derived by $T_{\mathrm{B}}=T_{\mathrm{A}}^{*} / \eta_{\mathrm{mb}}$ with the mainbeam efficiency $\eta_{\mathrm{mb}}(=0.74)$. The $1 \sigma$ noise levels of the grid-mapping data (in $\left.T_{\mathrm{B}}\right)$ are $17 \mathrm{mK}$ and $15 \mathrm{mK}$ for the ${ }^{12} \mathrm{CO}$ and ${ }^{13} \mathrm{CO}$ maps, respectively. The noise levels of central pointings with the long integrations are $11 \mathrm{mK}$ for the ${ }^{12} \mathrm{CO}$ and $3 \mathrm{mK}$ for the ${ }^{13} \mathrm{CO}$.

\subsection{Joint Deconvolution of SMA and SMT Data}

We used MIRIAD to combine the SMA interferometric and SMT single-dish data following the procedure described by Koda et al. (2011). Visibilities from the SMT single-dish images are generated by (1) deconvolving the SMT maps with their Gaussian beams (FWHMs of $\sim 33^{\prime \prime}$ and $\sim 34^{\prime \prime}$ for ${ }^{12} \mathrm{CO}$ and ${ }^{13} \mathrm{CO}$ frequencies, respectively), (2) attenuating these deconvolved SMT images with the SMA primary beams (FWHMs of $\sim 55^{\prime \prime}$ and $\sim 57^{\prime \prime}$, respectively), and (3) generating visibilities from the above (SMA primary beam attenuated) maps. We set the integration time per visibility equivalent to be the one per SMA visibility. The number of SMT visibilities was $3 \times 10^{4}$; varying this number within a factor of a few does not change the final image as far as the uniform weighting is employed. These SMT visibilities are combined with the SMA visibilities from all configurations and Fourier-transformed to dirty images by the MIRIAD's invert task. Figure1 shows the flux consistency of the visibility data from the SMT and the different configurations of SMA.

After the deconvolution process with the MIRIAD's clean task, we restored the clean components with the Gaussian beams that have the same solid angles as their corresponding dirty beams. Koda et al. (2011) emphasized the importance of this process for conserving the total flux, in particular, when sources with extended low level emission are involved. Conventionally in a restored image, the clean components are convolved with a synthesized beam (defined as a Gaussian function fitting the main lobe of the dirty beam), and the residuals (in units of the dirty beam solid angle) are added back. Departure of the dirty beam from the Gaussian shape leads to

\footnotetext{
${ }^{3}$ GILDAS is developed and distributed by the Observatoire de Grenoble and IRAM.
} 
inconsistency in the beam solid angle between the dirty beam and the derived synthesized beam, resulting in an artificial mismatch of the total flux. The conventional method works well in the cases of compact sources, where the cleaning process results in negligible residuals. In our case, because of the extended nature of the CSE and the non-Gaussian shape of the dirty beam, we defined new Gaussian beams having the same solid angles as the dirty beams, ensuring the total fluxes to be conserved after cleaning and restoring the images. The PAs and beam axis ratios are set to the ones for the main lobes of dirty beams.

Finally, the combined images are corrected for the SMA primary beam attenuation. More detailed description for combining the single-dish and interferometer data can be found in Koda et al. (2011). The final images of ${ }^{12} \mathrm{CO}$ and ${ }^{13} \mathrm{CO}$ have the beam sizes of $2^{\prime \prime} .14 \times 1^{\prime \prime} .74$ and 2 .'.23 $\times 11^{\prime \prime} .79$, respectively, with the PA of $37^{\circ}$. The noise levels are $25 \mathrm{mJy}$ beam ${ }^{-1}$ (150 mK) for the ${ }^{12} \mathrm{CO}$ map and $22 \mathrm{mJy}_{\text {beam }}{ }^{-1}(140 \mathrm{mK})$ for the ${ }^{13} \mathrm{CO}$ map.

\section{FEATURES OF OVERALL STRUCTURE}

\subsection{Continuum Emission: Position and Flux of the Radio Photosphere}

The position and flux of the continuum are determined by the uvfit task of MIRIAD. In both $226.2 \mathrm{GHz}$ and $350.6 \mathrm{GHz}$ continuum, the visibility amplitudes are fairly flat in the $u v$ distance (i.e., projected baseline length in wavelengths) range of $40-400 \mathrm{k} \lambda$ (see Figure2), implying a spatially unresolved source. In this $u v$ distance range, we have tried to find the best point, Gaussian, or disk source model, resulting in the flux and positional offset almost independent on the models. Moreover, the resulting Gaussian and disk sizes are comparable to the sizes in our trial fit for a phase calibrator, essentially a point source; therefore we adopt the point source model for the visibility data at $40-400 \mathrm{k} \lambda$.

The $226.2 \mathrm{GHz}$ continuum visibility amplitudes from the subcompact and compact configurations of SMA $(4-50 \mathrm{k} \lambda)$ are higher and increase toward the shorter baselines. The visibility data from all SMA configurations are best fit with a two-component model in a combination of one point source and one extended component as denoted by a solid curve in Figure2 (see Table 3). The residual image of the two-component model indeed shows a random noise distribution (Figure $3(\mathrm{~b})$ ), in contrast to the one-component model where unexpected structures remain in the residuals (Figure $3(\mathrm{c})-(\mathrm{d}))$. Given the uncertainty of visibility data, it is currently difficult to differentiate the Gaussian and disk models for the extended component.

Figure 4 presents the comparison of the SMA continuum flux at $226.2 \mathrm{GHz}$ and $350.6 \mathrm{GHz}$ with various measurements known in the literature at different frequencies. The $226.2 \mathrm{GHz}$ and $350.6 \mathrm{GHz}$ flux of the unresolved continuum component (together with a $36.5 \mathrm{GHz}$ continuum flux from another subarcsec-resolution observation with the JVLA) is described by a spectral index $n=-2$, where $n$ is defined by $F_{\nu} \propto \lambda^{n}$. This spectral index is expected for thermal blackbody 
radiation in the Rayleigh-Jeans limit. The compact nature and the spectral index close to -2 in the millimeter and centimeter wavelengths may indicate that the unresolved component of the SMA continuum emerges from a "radio photosphere" surrounding the stellar (optical) photosphere, which is introduced by Reid \& Menten (1997) for long-period variables. Reid \& Menten showed that the radio photosphere is about twice the size of the optical photosphere.

With an effective temperature $(\sim 2800 \mathrm{~K})$ of CIT 6 from the observed Na I D line at $5893 \AA$ (Cohen 1979) and its bolometric luminosity $\sim 10^{4} L_{\odot}$ from the integrated infrared emission (Loup et al. 1993), the Stefan-Boltzmann law $L_{*} / L_{\odot}=\left(R_{*} / R_{\odot}\right)^{2}\left(T_{*} / T_{\odot}\right)^{4}$ implies the stellar (optical) photosphere radius $R_{*} \sim 2 \mathrm{AU}$. Adopting the radio photosphere to be twice the size of the stellar photosphere (Reid \& Menten 1997), we applied a diameter of 8 AU (20 mas) and the corresponding radio photosphere brightness temperature of $2000 \mathrm{~K}$ for a black body curve drawn by a solid line in Figure 4. This line is consistent with the SMA measurements for the unresolved continuum source. The radio photosphere size of CIT 6 is comparable to that of IRC +10216 (11 AU) observed as a resolved disk at $43.3 \mathrm{GHz}$ continuum (Menten et al. 2012).

The data from infrared to centimeter wavelengths are fitted to a spectral index of -2.4 , confirming the result of Marshall et al. (1992) via a fit over a wide wavelength range $(0.5 \mu \mathrm{m}-1 \mathrm{~mm})$. It presents the excess thermal emission from circumstellar dusts. The upper point at $226.2 \mathrm{GHz}$ $(1.3 \mathrm{~mm})$ in Figure 4 is the total flux of the two-component (point source and Gaussian) model given in Table 3 . If the dashed line fit provides a rough estimate for the total flux at $226.2 \mathrm{GHz}$, albeit not obvious with the large deviation of the accumulated data, the extended continuum component of our SMA observations $\left(34.5 \mathrm{mJy}, \mathrm{FWHM} \lesssim 4^{\prime \prime}\right)$ recovers about $50 \%$ of the total thermal dust emission. There might exist more extended dust emission, missed in the SMA interferometric observations.

The coordinate center in this paper corresponds to right ascension (RA) at $10^{\mathrm{h}} 16^{\mathrm{m}} 02^{\mathrm{s}} .259$ and declination (Dec) at $+30^{\circ} 34^{\prime} 19^{\prime \prime}$. 18 in J2000 coordinates, derived from the visibility fitting of the unresolved continuum emission of CIT 6 at $226.2 \mathrm{GHz}$. Figure 5 shows the comparison of this position at the epoch 2013.1 with the optical, HCN maser, and $90.7 \mathrm{GHz}$ continuum positions of CIT 6 at the epochs 1955.3, 1988.1, and 1995.2 (Claussen et al. 1987; Carlstrom et al. 1990; Lindqvist et al. 2000). From the center positions in the four measurements, we calculate the linear proper motion

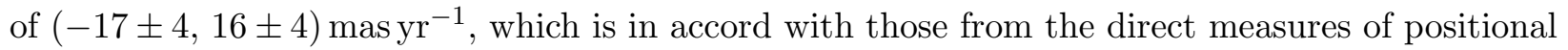
shifts with respect to reference stars in optical and infrared images; $(-16 \pm 10,14 \pm 10)$ mas yr$^{-1}$ (Monnier et al. 2000), and (-25 $\pm 10,19 \pm 10)^{m_{a s ~ y r}}{ }^{-1}$ (Röser et al. 2008). Therefore, CIT 6 is moving to the northwest direction with a projected speed of $\sim 44 \mathrm{~km} \mathrm{~s}^{-1}$, which is also consistent with the speed derived from an astrospheric feature at $\sim 15^{\prime}-18^{\prime}$ in far-ultraviolet images (Menten et al. 2012, Sahai \& Mack-Crane 2014). 


\subsection{Spectral Profile}

Figure 6 illustrates the spectral profiles of ${ }^{12} \mathrm{CO} J=2-1$ and ${ }^{13} \mathrm{CO} J=2-1$ lines varying with integration radius. The ${ }^{12} \mathrm{CO}$ spectra have a red-skewed doubly-peaked profile when integrated over the central region $\left(r \leq 1^{\prime \prime}\right)$ as shown in panel (a), a flat-topped profile over $\leq 5^{\prime \prime}$ region in (b), and approximately parabolic profiles over larger $\sim 33^{\prime \prime}$ and $\sim 2.5$ regions in $(\mathrm{c})$ and $(\mathrm{d})$. These parabolic and flat-topped shapes of ${ }^{12} \mathrm{CO}$ line profiles are typical for an optically thick source when it is spatially unresolved and resolved, respectively (Morris 1975).

In contrast, the ${ }^{13} \mathrm{CO}$ spectral profiles have double peak features regardless of the integration areas, although the spectrum in (d) integrated over the entire SMT mapping area may be also interpreted as a flat-topped profile with the given noise. According to Morris (1975), an optically thin source produces a double peak shape in the spectral profile when it is spatially resolved, and a flat-topped profile when unresolved; hence, the ${ }^{13} \mathrm{CO}$ line emitting region is likely similar to, or smaller than, the SMT mapping area (i.e., the integrated area for (d) panel). The ${ }^{13} \mathrm{CO}$ envelope is generally smaller than the ${ }^{12} \mathrm{CO}$ envelope because of the self-shielding capability of the abundant ${ }^{12} \mathrm{CO}$ molecules against photodissociation by interstellar ultraviolet radiation field. Therefore, considering the nearly flat-topped shape of ${ }^{13} \mathrm{CO}$ line together with the parabolic shape of ${ }^{12} \mathrm{CO}$ line, our SMT mapping area $(\sim 2.5 \times 2 \cdot 5)$ likely covers most of, if not all of, the entire CSE of CIT 6 in the ${ }^{12} \mathrm{CO} J=2-1$ and ${ }^{13} \mathrm{CO} J=2-1$ line emission.

We define the systemic velocity $\left(v_{\text {sys }}=-2 \mathrm{~km} \mathrm{~s}^{-1}\right)$ as the middle of two peaks of the ${ }^{13} \mathrm{CO}$ $J=2-1$ spectrum from the central $1^{\prime \prime}$ region, presented in (a) panel. The velocity $v_{z}$ hereafter represents the velocity with respect to the systemic velocity $\left(v_{z}=v_{\mathrm{LSR}}-v_{\mathrm{sys}}\right)$. The ${ }^{13} \mathrm{CO}$ spectrum in the central $1^{\prime \prime}$ region is sharply doubly peaked at $v_{z}= \pm 16 \mathrm{~km} \mathrm{~s}^{-1}$, beyond which the intensity levels quickly drop to zero within $2 \mathrm{~km} \mathrm{~s}^{-1}$. The outer parts of ${ }^{13} \mathrm{CO}$ emission seem to have an extra velocity component around $-10 \mathrm{~km} \mathrm{~s}^{-1}$, as seen in (b) panel, in a comparable brightness temperature level with that of the $-16 \mathrm{~km} \mathrm{~s}^{-1}$ component. In (c) and (d) panels, this component predominates over the $-16 \mathrm{~km} \mathrm{~s}^{-1}$ component that appears as a broader shelf in the blueshifted velocity range. In contrast, the red peak velocity does not change much with the integration radius.

The ${ }^{12} \mathrm{CO} J=2-1$ spectral asymmetry depends on the integration area. In Figure6(a), the central region $\left(r \leq 1^{\prime \prime}\right)$ has a red-skewed doubly peaked spectrum with the redshifted peak (at $v_{z}=$ $14 \mathrm{~km} \mathrm{~s}^{-1}$ ) about $30 \%$ brighter than the blueshifted peak (at $-8 \mathrm{~km} \mathrm{~s}^{-1}$ ). In order to quantify the spectral profile asymmetries varying with the integration area, we define the fraction of asymmetry as the emission excess of the positive velocity component (at 0 to $16 \mathrm{~km} \mathrm{~s}^{-1}$ ) beyond the negative velocity component (at -16 to $0 \mathrm{~km} \mathrm{~s}^{-1}$ ). In the central $1^{\prime \prime}$ region shown in (a), the positive velocity emission is about $40 \%$ brighter than the negative velocity emission, i.e., the fraction of asymmetry is $40 \%$. As the integration area increases, the fraction of asymmetry is reduced to $30 \%$ in the (nearly) flat-topped profile in $r \leq 5^{\prime \prime}$ region shown in (b), and to $10 \%$ and $5 \%$ in (c) and (d), respectively. This decrease of the fraction of asymmetry with the integration radius implies that the source of spectral asymmetry is placed in the central region of the ${ }^{12} \mathrm{CO}$ line emission. For comparison, the 
fractions of asymmetry of the ${ }^{13} \mathrm{CO} J=2-1$ line spectra are within $\pm 5 \%$ regardless of integration area.

\subsection{Averaged Radial Distribution}

Considering the CSE of an AGB star having radial flows with a nearly constant velocity, the channel at the expansion velocity exhibits emission integrated along the line of sight. Hence, it is subject to an optically thick condition. On the other hand, the optical depth at the systemic velocity channel, exhibiting the emission from the plane of the sky, is relatively small. Therefore, in Figure 7, we use the systemic velocity channel to compare the radial profiles of the ${ }^{12} \mathrm{CO} J=2-1$ and ${ }^{13} \mathrm{CO} J=2-1$ brightness temperatures averaged over azimuthal angles.

In order to examine the brightness distribution from small to large scales, we generate maps for four different combinations of SMA and SMT data: SMA-only (yellow; beam size $\sim 0$ '”5), joint of SMA and SMT (black; $\sim 2^{\prime \prime}$ ), joint of SMA subcompact and compact configuration with SMT (blue; $\sim 6^{\prime \prime}$ ), and SMT-only (red; $\sim 33^{\prime \prime}$ ). All four curves for ${ }^{12} \mathrm{CO} J=2-1$ are consistent with each other (except for the SMA-only profile beyond 10"; see next paragraph), following a monotonically decreasing curve from $r \sim 0$ '! 2 to $60^{\prime \prime}$. For the $\sim 20$ times weaker ${ }^{13} \mathrm{CO} J=2-1$ emission, the trend can be found by connecting the profiles from different configurations at sufficient signal-to-noise. The ${ }^{12} \mathrm{CO}$ profile changes slope (in logarithmic scale) smoothly from $\sim-0.7$ for the inner part $\left(r \lesssim 5^{\prime \prime}\right)$ to $\sim-2.0$ beyond $5^{\prime \prime}$. On the other hand, the ${ }^{13} \mathrm{CO}$ profile keeps the shallow slope $\sim-0.7$ up to the radius $\sim 20^{\prime \prime}$ and then drops with a markedly steeper slope $\sim-3.0$. As a result, the ${ }^{12} \mathrm{CO}$ and ${ }^{13} \mathrm{CO} J=2-1$ line ratio is $\sim 30$ in the inner $5^{\prime \prime}$ region, $\sim 10$ at $\sim 20^{\prime \prime}$, and $\sim 20$ beyond $30^{\prime \prime}$.

The only significant deviation from the rest configuration data occurs in the SMA-only profile, dramatically dropping beyond its maximum recoverable scale $\left(r \sim 10^{\prime \prime}\right)$ due to the finite minimum projected baseline $(\sim 5 \mathrm{~m})$. Despite such missing flux in the SMA interferometry beyond the maximum recoverable scale, the minimum baseline of the SMA-only data was sufficiently short so that the resulting brightness temperature reduction $(<2 \mathrm{~K})$ does not significantly change the profile within $\sim 10^{\prime \prime}$. Indeed, the convolution of the SMA-only map with a $23^{\prime \prime}$-sized Gaussian beam yields the peak and integrated brightness temperatures of $3.74 \mathrm{~K}$ (at $v_{z}=0 \mathrm{~km} \mathrm{~s}^{-1}$ ) and $98.0 \mathrm{~K} \mathrm{~km} \mathrm{~s}^{-1}$, essentially identical with the values from a $23^{\prime \prime}$-beam single-dish observation $(3.72 \mathrm{~K}$ and $97.3 \mathrm{~K} \mathrm{~km} \mathrm{~s}^{-1}$, Olofsson et al. 1993). The SMA-related profiles (yellow, black, and blue) have large error bars beyond the SMA primary beam radius $\sim 30^{\prime \prime}$. 


\section{FINE STRUCTURE}

\subsection{Spiral-shell Pattern}

Figure 8 displays the ${ }^{12} \mathrm{CO} J=2-1$ channel map of CIT 6 from the SMA observations with a $\sim 0^{\prime \prime} .5$ resolution for a channel velocity width of $5 \mathrm{~km} \mathrm{~s}^{-1}$ (in pseudo blue color). For comparison, it is overlaid by the $\mathrm{HC}_{3} \mathrm{~N} J=4-3$ map (in pseudo red color) taken with the JVLA (Claussen et al. 2011). The broken-shells of the $\mathrm{HC}_{3} \mathrm{~N}$ emission for CIT 6 were used in the previous spiral-shell modeling by Kim et al. (2013). The central velocity channels (middle row panels) indeed reveal spiral patterns in ${ }^{12} \mathrm{CO}$, and the patterns at higher velocities (top and bottom panels) are rather ring-like. Both of these features agree well with the spiral-shell geometry (see e.g., Figure 8 of Kim \& Taam 2012b) implying the existence of the companion to CIT 6 and hence diagnostics for the orbital motion of the binary system (Kim et al. 2013).

A single spiral pattern is particularly discerned in the $v_{z}=5 \mathrm{~km} \mathrm{~s}^{-1}$ channel (middle right panel of Figure 8). The pattern has a shape rolling inward in the clockwise sense, indicating the orientation of binary orbital motion. Inner two windings are apparent in the ${ }^{12} \mathrm{CO}$ map with the brightness level over $20 \sigma$. On the other hand, the pattern beyond $\sim 5^{\prime \prime}$ is rather sporadic. The arm at $\sim 8^{\prime \prime}$ to the west is perceptible at $\sim 6 \sigma$ with a higher arm-interarm contrast than in other directions, caused by a reduction of the interarm brightness level (see below).

Figure 9 displays the radial profiles of the ${ }^{12} \mathrm{CO}$ line emission at the systemic velocity channel $\left(v_{z}=0 \mathrm{~km} \mathrm{~s}^{-1}\right)$. Each colored curve is defined along the noted PA by azimuthal average with the width of $20^{\circ}$, and the bottom profile is averaged over $360^{\circ}$. The arms are partially resolved with the SMA beam size $\sim 0$ ".5, which would form otherwise almost monotonically decreasing curves (as in Figure 7, the profile for the SMA-SMT combined image with a larger $\sim 2^{\prime \prime}$-sized beam). The radii of local peak positions differ with $\mathrm{PA}$, indicating the non-spherical nature as expected in a spiral model. The emission in the spiral arm appears as $20 \%$ fluctuation of total emission, and the peak values in the arm regions exceed the adjacent interarm values by a factor of $\sim 1.5$ to $\sim 3$.

Except for the west side (PA of $270^{\circ}$ ), no systematic difference is found in the shape of directional profiles, unlike in close binary systems presenting the envelope flattening onto the orbital plane (see Huggins et al. 2009; Mauron et al. 2013). The brightnesses decrease to $3 \sigma$ (color-coded dotted lines in Figure 9p at $r \sim 9^{\prime \prime}$ except for the profile to the west, where the brightness reaches $3 \sigma$ at a smaller radius $r \sim 5^{\prime \prime}$. The overall profile to the west is $25 \%$ steeper than in all the other directions. This deficiency of the ${ }^{12} \mathrm{CO}$ line emission to the west probably indicates a lower mass ejection along this direction due to, e.g., anisotropic dust formation near the stellar surface, or non-constant orbital speed of the star.

The position-velocity $(\mathrm{P}-\mathrm{V})$ diagrams $\mathrm{S}^{4}$ in Figure $10(\mathrm{a})-(\mathrm{b})$ better exhibit the pattern in a con-

\footnotetext{
4 Kim et al. (2013) suggested the PA of $10^{\circ}$ as the intersection between the plane of the sky with the orbital plane of the binary stars, along which the P-V diagram is the most symmetric and perpendicular to which $\left(\mathrm{PA}\right.$ of $\left.280^{\circ}\right)$
} 
tinuous and definitive shape. The arm patterns in Figure 10(a) are rather regularly arranged as

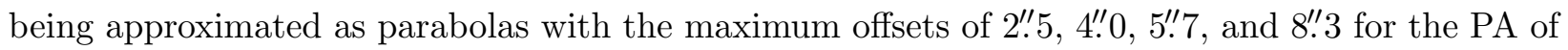
$10^{\circ}$ (positive offset in the panel) with intervals of $1^{\prime \prime} .5,1^{\prime \prime} .7$, and $2^{\prime \prime} 6$; for the PA of $190^{\circ}$ (negative

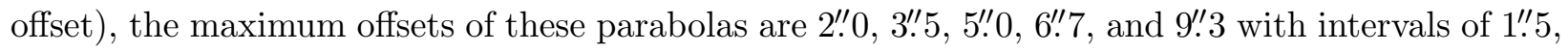
1". $5,1^{\prime \prime} .7$, and 2.' 6 . The observed P-V diagram patterns tend to have an arm interval of $\sim 1$ 1.5 in the inner $\sim 7^{\prime \prime}$ region, but have a larger interval of $2^{\prime \prime} .5$ between the observed outermost arms located at $\sim 6^{\prime \prime}-9^{\prime \prime}$. This change of arm interval can be due to a temporal decrease of the wind velocity.

\subsection{Emission Peak Position with Channel Velocity}

Upon a close inspection of the zoomed-in channel maps for the ${ }^{12} \mathrm{CO}$ emission (Figure 11), the shape of emitting region is not circular and varies with channels. We further investigate the positional change of channel peaks by fitting the observed offsets along the axes of RA and Dec with sinusoidal functions in the form of $f=a_{0}+a_{1} \sin \left(2 \pi\left(v_{z}-a_{3}\right) / a_{2}\right)$, as shown in Figure 12(a)(b). The parameter $a_{0}$ represents the average emission peak position, $a_{1}$ the scale of maximum positional shift, and $a_{2}$ the channel velocity scale for which the positional variation of emission peaks occurs. Here, we focus on the velocity range between -13 and $16 \mathrm{~km} \mathrm{~s}^{-1}$. The rapid change of peak positions at the high-end velocities (related to a bipolar outflow) is discussed in Section 4.3 .

These separately fitted sinusoidal functions for RA and Dec both have the same $a_{2}$ parameter of $17.7 \mathrm{~km} \mathrm{~s}^{-1}$, indicating that the emission peak position varies periodically in the scale of the expansion velocity of the envelope. Because of the consistency of cycles in RA and Dec, the combined fitting results lead to a closed ellipse as a function of channel velocity as shown in Figure 12(c). It approximates an ellipse with an ellipticity $\sim 0.75$ and a major axis on the scale $\sim 0{ }^{\prime \prime} 15$ with the $\mathrm{PA} \sim-40^{\circ}$. The peak positions of channels at $\sim-8 \mathrm{~km} \mathrm{~s}^{-1}$ and $\sim 10 \mathrm{~km} \mathrm{~s}^{-1}$ become closer to the continuum peak position, which we defined as the coordinate center. In average, the channel peaks of the ${ }^{12} \mathrm{CO} J=2-1$ emission are located at the northwest side of the

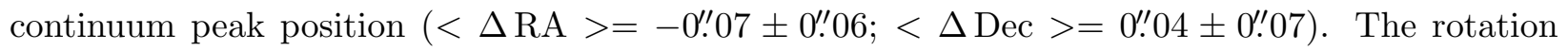
of peak positions along the channels were also reported by Lindqvist et al. (2000) for the HCN $J=1-0, \mathrm{HNC} J=1-0, \mathrm{HC}_{3} \mathrm{~N} J=10-9$, and SiS $J=5-4$ line emission over larger scales $\left(\sim 00^{\prime \prime} 5-2^{\prime \prime}\right)$; the loci of their channel peaks could be connected to a curve elongated in the northwest-southeast direction across the line profile, similar to the PA of ellipse in our fitting result of the ${ }^{12} \mathrm{CO}$ peak positions.

The positional measurement uncertainty is given by the largest value amongst (1) the beam size divided by the peak signal-to-noise ratio $\left(\lesssim 00^{\prime \prime} 01\right.$ in most channels; it increases to $\sim 00^{\prime \prime} 06$ at the line edge channel), (2) the positional (or phase) uncertainty of the gain calibrators derived by the same method experiments $(<0 . \prime 03)$, and (3) the image pixel size $\left(0^{\prime \prime} \cdot 03\right)$. However, these provide a

the $\mathrm{P}-\mathrm{V}$ diagram is the most asymmetric. 
lower limit of the positional uncertainty as the ${ }^{12} \mathrm{CO}$ emission is extended. A firm conclusion on the spatial change of the emission peak (in a rotating sense) with velocity and its relationship with the binary motion in an inclined orbital plane are the subject of higher spatial and spectroscopic resolution observations.

\subsection{Bipolar Outflow}

The ${ }^{12} \mathrm{CO}$ spectrum in Figure 6(a) for the central $1^{\prime \prime}$ region exhibits a small bump at $v_{z} \sim$ $-19 \mathrm{~km} \mathrm{~s}^{-1}$, distinguished from the main line by a valley at $v_{z}=-16 \mathrm{kms}^{-1}$. Such a feature is also found in $\mathrm{P}-\mathrm{V}$ diagrams, appearing as an island located at $-21 \lesssim v_{z} \lesssim-17 \mathrm{~km} \mathrm{~s}^{-1}$ (see Figure 10(a)-(b)). This component contributes about $1.4 \%$ in the total flux.

Other interferometric observations showed the blue bump feature in several pPNe (e.g., IK Tau, $\chi$ Cyg, IRAS 20028+3910, and IRAS 23321+6545 in Figures 4, 22, and 23 of Castro-Carrizo et al. 2010), where fast bipolar outflow structures are well developed. These spectral features were interpreted as an indication of heating due to shock interaction between the inner fast collimated winds and the outer slow nearly spherical winds ejected during the AGB phase. The emission from the hot shocked region is vulnerable to self-absorption by the surrounding cooler medium. The blue bump feature, in addition to the depressed blue peak below the red peak in Figure6, can be also induced in a perfectly spherical wind due to partial self-absorption of the central warmer emission by the outer cooler envelope (Morris et al. 1985). Self-absorption was introduced by both Morris et al. (1985) and Castro-Carrizo et al. (2010) in explaining the blue bump features, but the geometry and physical mechanism of the hot inner source are different from each other.

As clearly seen in Figure 13, the observed blue bump of CIT 6 originates from a bipolar outflow. Figure 13 is the same as the highest velocity channels in Figure 8 , but in blue and red contours for the blueshifted $\left(-19 \mathrm{~km} \mathrm{~s}^{-1}\right)$ and redshifted $\left(19 \mathrm{~km} \mathrm{~s}^{-1}\right)$ components, respectively. For comparison, the emission peak at the systemic velocity is also plotted in green contours, showing its position significantly close to the continuum peak position (gray-scaled image). In contrast, the high-velocity emission peaks are definitely offset from the continuum peak position to the northeast-southwest direction. With the directional similarity, we consider these high velocity components as the gaseous counterpart of the near-infrared bipolar nebula (Schmidt et al. 2002).

The $-19 \mathrm{~km} \mathrm{~s}^{-1}$ component has an elongated shape to the northeast. It is distributed in a conical shape about the PA of $55^{\circ}$, which is consistent with the PA of the near-infrared reflection nebula $\left(0^{\circ}-80^{\circ}\right)$ shown in Schmidt et al. (2002). The size $\left(\sim 3^{\prime \prime}\right.$ at $\left.3 \sigma\right)$ of this component is comparable to the size of the near-infrared bipolar nebula. Its conical shape has the vertex close to, but slightly south of, the continuum peak position. Assuming that the continuum peak position coincides with the location of the visible red point source (the probable carbon star) in Monnier et al. (2000), the location of the visible blue point source (possibly the companion, $\lesssim 0^{\prime \prime} 2$ south of the red source) is marked by a cross symbol in Figure 13 . With the current angular resolution, 
it is difficult to distinguish whether the conical outflow originates from the carbon star or the companion.

The redshifted component is considerably smaller $\left(\sim 1.5^{\prime \prime}\right.$ at $\left.8 \sigma\right)$ than the blueshifted component. The redshifted component at $\sim 19 \mathrm{~km} \mathrm{~s}^{-1}$ extends to the south or southeast in Figure 13 . The central spectrum in Figure6(a) does not show any special feature at $\sim 19 \mathrm{~km} \mathrm{~s}^{-1}$, unlike the blueshifted component appears as a small bump. However, Figure 11 and 12 show that the position of emission peak in each channel beyond $16 \mathrm{~km} \mathrm{~s}^{-1}$ gradually shifts to the southeast as the velocity increases. This shift is noticeable because the emission peak of each channel remains within the central $0^{\prime \prime} 2$ regime in most of velocity range $\left(-13\right.$ to $\left.16 \mathrm{~km} \mathrm{~s}^{-1}\right)$. The $19 \mathrm{~km} \mathrm{~s}^{-1}$ component appears to consist of two features, one in a PA of $190^{\circ}$ and the other in $150^{\circ}$. This is most evident for the red contour corresponding to $12 \sigma$. The component along the $\mathrm{PA}$ of $190^{\circ}$ is approximately in the direction opposite to the blue component. The presence of red component along the PA of $150^{\circ}$ implies multipolar nature, together with a discrete blue component at $8 \sigma$ level in the opposite (northwest) direction.

We note a cometary-shaped silicate feature at $9.7 \mu \mathrm{m}$ (Lagadec et al. 2005) along the PA of $\sim 230^{\circ}$ with a large extension up to $7^{\prime \prime}$ (at $1 \%$ of the peak intensity), which is exactly on the opposite side of our $-19 \mathrm{~km} \mathrm{~s}^{-1}$ component. Currently, however, there is no strong evidence to further relate the $9.7 \mu \mathrm{m}$ feature to the redshifted component of the bipolar outflow.

\section{DISCUSSION}

\subsection{One-Dimensional Radiative Transfer Model}

We have performed simple, one-dimensional, non-local thermodynamic equilibrium radiative transfer calculations using the SPARX code 5 to understand the overall distribution of the ${ }^{12} \mathrm{CO}$ and ${ }^{13} \mathrm{CO}$ molecules in the CSE of CIT 6 . Since our focus here is on the overall CSE distribution, the observed fine structures (as described in Section 4) are ignored in the modeling; see Kim et al. (2013) for a spiral-shell model for CIT 6, characterizing the $\mathrm{HC}_{3} \mathrm{~N} J=4-3$ pattern in channel maps. In Figures 6 and 7, we present a simple model for an AGB envelope expanding with a constant expansion velocity $V_{w}=18 \mathrm{~km} \mathrm{~s}^{-1}$, a mass loss rate $\dot{M}=8 \times 10^{-6} M_{\odot} \mathrm{yr}^{-1}$, a temperature distribution $T(r)=2000 \mathrm{~K}(\mathrm{r} / 4 \mathrm{AU})^{-0.7}$, a stochastic velocity of $1 \mathrm{~km} \mathrm{~s}^{-1}$, constant fractional abundances of molecules $\left(f_{12} \mathrm{CO}=1 \times 10^{-3}\right.$ and $\left.f_{13} \mathrm{CO}=2 \times 10^{-5}\right)$, and a distance of $400 \mathrm{pc}$.

This model reproduces reasonably well the average radial profile of the observed ${ }^{12} \mathrm{CO} J=2-1$ (Figure7, green curve) and its spectra in different length scales (Figure6, dashed line), providing

\footnotetext{
${ }^{5}$ SPARX (Simulation Package for Astrophysical Radiative Transfer) is a software package for calculating molecular excitation and radiative transfer of dust continuum and molecular lines at millimeter/submillimeter wavelengths (Liu et al., in prep.).
} 
some insights to physical conditions of the overall CSE of CIT 6 . The optical depth of the ${ }^{12} \mathrm{CO}$ $J=2-1$ model at the systemic velocity is low $(\sim 0.5)$ at the center, increases to $\sim 2$ at $r \sim 10^{\prime \prime}$, and decreases back to $\sim 0.5$ at $r \sim 40^{\prime \prime}$. On the other hand, the expansion velocity channel has the large optical depth greater than 4 for almost all emitting regions with the peak value over 10 at $r \sim 4^{\prime \prime}$. The over-predicted flux in Figure 6(d) may indicate that the ${ }^{12} \mathrm{CO}$ abundance in the outermost region is lower than in the inner region possibly because of photodissociation due to interstellar ultraviolet photons. This interpretation is consistent with the radius at which the abundance drops to half of its photospheric value derived to be $\sim 30^{\prime \prime}$ by e.g., Schöier \& Olofsson (2001), based on Mamon et al. (1988).

The radial profile of ${ }^{13} \mathrm{CO} J=2-1$, however, is not achieved by merely changing the single molecular fractional abundance with the same fixed mass loss rate and temperature profile as in ${ }^{12} \mathrm{CO}$ model. The ${ }^{13} \mathrm{CO}$ modeled and observed spectra are not too far off in the inner part (Figure6(a)-(b)); but the outer envelope of CIT 6 contains significantly more ${ }^{13} \mathrm{CO}$ emission compared to the model (Figure 6(c)-(d)). In fact, as seen in Figure 7, the ${ }^{13} \mathrm{CO}$ radial profile of CIT 6 is relatively flatter and more attributed by extended component than in ${ }^{12} \mathrm{CO}$. The significant difference in slopes of the ${ }^{12} \mathrm{CO}$ and ${ }^{13} \mathrm{CO}$ radial profiles may imply a variation of their abundance ratio with radius.

The ${ }^{12} \mathrm{CO} /{ }^{13} \mathrm{CO}$ abundance ratio 50 in the presented model is somewhat larger than the value $\lesssim 35$ in the literature (Milam et al. 2009 , and reference therein). Our choice for the ${ }^{13} \mathrm{CO}$ fractional abundance results from matching the brightness temperatures in the inner region closely the observed values, at the expense of the outer region. We have checked that the modeled ${ }^{13} \mathrm{CO}$ spectra with a ratio $\sim 30$ can reproduce the SMT single-dish observational data (i.e., for Figure6(c)(d)), consistent with the earlier models by e.g., Milam et al. (2009), but resulting in a mismatch in the inner envelope. It may indicate that ${ }^{12} \mathrm{CO} /{ }^{13} \mathrm{CO}$ abundance ratio varies with radius.

\subsection{Comparison with $\mathrm{HC}_{3} \mathrm{~N}$}

Considering both the ${ }^{12} \mathrm{CO} J=2-1$ and $\mathrm{HC}_{3} \mathrm{~N} J=4-3$ high resolution maps together, four to five windings of the spiral-shell pattern are found. In terms of their location, there are close agreements between the ${ }^{12} \mathrm{CO}$ and $\mathrm{HC}_{3} \mathrm{~N}$ patterns manifested in the channel maps (Figure 8) and the $\mathrm{P}-\mathrm{V}$ diagrams (Figure 10). For example, to the west where the $\mathrm{HC}_{3} \mathrm{~N}$ pattern is broken, the ${ }^{12} \mathrm{CO}$ pattern is present and smoothly connects the $\mathrm{HC}_{3} \mathrm{~N}$ segments from the north to the south. Figure 10 (a) and (c) show the ${ }^{12} \mathrm{CO}$ and $\mathrm{HC}_{3} \mathrm{~N}$ patterns along the $\mathrm{PA}$ of $10^{\circ}$, and their overlay in Figure 10 (e) demonstrates their coincidence. The local peak positions are marked by open circles (blue for ${ }^{12} \mathrm{CO}$; red for $\mathrm{HC}_{3} \mathrm{~N}$ ), illustrating their good match in the $\mathrm{P}-\mathrm{V}$ diagram. In the bottom panels of Figure 10 along the $\mathrm{PA}$ of $280^{\circ}$, the $\mathrm{HC}_{3} \mathrm{~N}$ pattern in (d) is prominent in the outer shells, while the ${ }^{12} \mathrm{CO}$ emission in (b) is centralized and the outer pattern fading away. However, their overlay in (f) again shows a good correlation. 
Albeit there is a good correlation in the pattern locations, some differences can be found between the ${ }^{12} \mathrm{CO} J=2-1$ and $\mathrm{HC}_{3} \mathrm{~N} J=4-3$ maps. The ${ }^{12} \mathrm{CO}$ emission is omnipresent in the CSE of CIT 6, while the $\mathrm{HC}_{3} \mathrm{~N}$ line emission is absent toward the center and west sides (see Figure8, e.g., the panels for $v_{z}=0 \mathrm{~km} \mathrm{~s}^{-1}$ and $5 \mathrm{~km} \mathrm{~s}^{-1}$ ). The ${ }^{12} \mathrm{CO}$ map exclusively reveals the first and second windings in the central $5^{\prime \prime}$ region, while their $\mathrm{HC}_{3} \mathrm{~N}$ counterparts are missed. The $\mathrm{P}-\mathrm{V}$ diagrams (Figure $10(\mathrm{a})-(\mathrm{b})$ ) further exhibit the richness of the ${ }^{12} \mathrm{CO}$ structure within the central $5^{\prime \prime}$, where $\mathrm{HC}_{3} \mathrm{~N}$ is lacking as shown in Figure 10 (c)-(d). The second and third ${ }^{12} \mathrm{CO}$ windings appear broken in the $\mathrm{HC}_{3} \mathrm{~N}$ map in the west side. The ${ }^{12} \mathrm{CO}$ structures appearing at $\sim 5^{\prime \prime}$ and $\sim 10^{\prime \prime}$ in Figure 10 (b) are completely missed in the corresponding $\mathrm{P}-\mathrm{V}$ diagram of $\mathrm{HC}_{3} \mathrm{~N}$, i.e., Figure $10(\mathrm{~d})$.

To the west side where the $\mathrm{HC}_{3} \mathrm{~N} J=4-3$ emission was absent, the ${ }^{12} \mathrm{CO} J=2-1$ arm pattern appears but its radial profile is steeper than in any other directions. It implies that the west side is indeed special in both molecules, probably indicating anisotropic mass loss. Nevertheless, the complete non-detection of the western part of spiral arm pattern in $\mathrm{HC}_{3} \mathrm{~N} J=4-3$ implies that the chemical effects on $\mathrm{HC}_{3} \mathrm{~N}$ may be not negligible.

The ${ }^{12} \mathrm{CO}$ emission is seen in both the compressed spiral arm and the interarm region, whereas the $\mathrm{HC}_{3} \mathrm{~N}$ emission seems to be mostly enhanced in the outer edge of the shock front as following. In the outer region where the $\mathrm{HC}_{3} \mathrm{~N}$ pattern is prominent $\left(\sim 3^{\prime \prime}\right.$ to $\left.10^{\prime \prime}\right)$, the spiral-shell pattern appears better in $\mathrm{HC}_{3} \mathrm{~N}$ with high arm-interarm contrasts. The $\mathrm{HC}_{3} \mathrm{~N}$ pattern segments are narrow (comparable to its synthesized beam size $\sim 00^{\prime \prime} 7$ ), while the ${ }^{12} \mathrm{CO}$ arm pattern is broader and the arm-interarm contrasts are smaller. In addition, the $\mathrm{HC}_{3} \mathrm{~N}$ emission peaks marked by red circles in Figure 10(e)-(f) tend to be located slightly outside of the ${ }^{12} \mathrm{CO}$ emission peaks (blue). All these features may indicate that $\mathrm{HC}_{3} \mathrm{~N}$ is sufficiently excited at the edge of the spiral arm region tracing the shock front.

The critical density for a molecular line transition, at which the emission induced by molecular collisions dominates over spontaneous emission, gives an approximate lower limit on the gas density in the emitting region of such line transition. The critical density of ${ }^{12} \mathrm{CO} J=2-1$ is nearly independent of temperature $\left(\sim 10^{4} \mathrm{~cm}^{-3}\right)$, while that of $\mathrm{HC}_{3} \mathrm{~N} J=4-3$ is higher $\left(>2.5 \times 10^{4} \mathrm{~cm}^{-3}\right.$ for $<100 \mathrm{~K})$ and drops with temperature ${ }^{6}$. It implies that the $\mathrm{HC}_{3} \mathrm{~N} J=4-3$ is a higher density tracer at the typical average temperatures of AGB CSEs $(<100 \mathrm{~K})$. In the radius range $\left(r \sim 3^{\prime \prime}-\right.$ $\left.10^{\prime \prime}\right)$ where the $\mathrm{HC}_{3} \mathrm{~N}$ pattern of CIT 6 is observed, a low interarm number density $\lesssim 2 \times 10^{3} \mathrm{~cm}^{-3}$ and gas temperature $\lesssim 40 \mathrm{~K}$ are expected (Dinh-V.-Trung \& Lim 2009; Kim et al. 2013). Therefore the lack of $\mathrm{HC}_{3} \mathrm{~N}$ line emission in the interarm region is reasonable. Within the spiral arm, a number density of $10^{4}-10^{6} \mathrm{~cm}^{-3}$ with a temperature of several hundreds Kelvins is possible Kim et al. 2013). Hence, molecular collisions are sufficient for exciting $\mathrm{HC}_{3} \mathrm{~N} J=4-3$, explaining the high arm-interarm contrast in the $\mathrm{HC}_{3} \mathrm{~N}$ map. On the other hand, the critical density of ${ }^{12} \mathrm{CO}$

\footnotetext{
${ }^{6}$ The critical densities are calculated based on the Leiden Atomic and Molecular Database (Schöier et al. 2005) with a simple two-level balance.
} 
$J=2-1$ is independent of gas temperature, making this emission well excited in both arm and interarm regions, and reducing the contrast between the arm and interarm regions.

\subsection{Signatures of Eccentric Orbit}

The systemic velocity channel interestingly shows a double spiral feature in the central $5^{\prime \prime}$ region, as shown in Figure 14 (see also the middle panel of Figure8 for a larger field), which was not generated in the previous hydrodynamic simulation assuming a constant wind velocity of $17 \mathrm{~km} \mathrm{~s}^{-1}$ Kim et al. 2013). A slower wind at the locus of the companion may facilitate generation of another spiral (see e.g., Figure 3 of Mohamed \& Podsiadlowski 2012) provided that the flow velocity is less than the escape velocity from the companion's gravitational potential. The inner double spirals are expected to merge together within one to two windings because of their different pattern propagation speeds. This merging seems to occur at around $4^{\prime \prime}$ in CIT 6 , leaving the onearmed spiral pattern in the outer part of Figure 8 (middle panel) as traced in the $\mathrm{HC}_{3} \mathrm{~N}$ and $\mathrm{CO}$ line emissions.

However, the projected binary separation of $\lesssim 80 \mathrm{AU}$ (Monnier et al. 2000) is far beyond several stellar radii, where dust grains form and stellar radiation pressures on them accelerate the CSE flow to the terminal velocity (Höfner 2007). We speculate that a highly eccentric orbit may allow the companion to pass the acceleration zone, possibly forming a double spiral in the inner part of the CSE of CIT 6. An orbit with the periastron closer than the acceleration zone radius (assuming $20 \mathrm{AU}$ ) and the apocenter at $r \gtrsim 80 \mathrm{AU}$ requires an eccentricity $\gtrsim 0.6$.

For an eccentric orbit, the orbital speed of a mass-losing star is fastest at the periastron. Therefore the accumulated mass ejection to this direction is lower than in other directions. The lack of ${ }^{12} \mathrm{CO}$ interarm emission to the west (along a PA of $270^{\circ}$ in Figure 9), closely resembles the column density map of a highly eccentric orbit model in $\mathrm{He}(2007$, see e.g., the model with an eccentricity of 0.8 ). The anisotropic mass loss due to the eccentric orbit alters the gas density particularly in the interarm region, which is similar to our finding from the ${ }^{12} \mathrm{CO}$ brightness radial profiles. In this case, the periastron would be on the west side.

Cernicharo et al. (2015) further employed a concept of periodic mass loss enhancement triggered by a close fly-by companion in their piston model, creating a more complex pattern on the CSE. If this strong tidal interaction model applies to CIT 6, the periastron, at which a larger mass loss could occur, would be on the opposite, east side leading to a higher average intensity. This situation is less likely for a wide binary like CIT 6, although the necessary binary separation for the strong tidal interaction between the stars is not well studied. 


\section{SUMMARY}

We have observed CIT 6 in all possible (subcompact, compact, extended, and very extended) array configurations of the SMA from 2013 January to 2014 March, and combined with the SMT mapping observations on 2015 January. Our findings from the ${ }^{12} \mathrm{CO} J=2-1,{ }^{13} \mathrm{CO} J=2-1$, and continuum emission are summarized as follows:

- CIT 6's continuum position at the epoch 2013.1 is $(\mathrm{RA}, \mathrm{Dec})=\left(10^{\mathrm{h}} 16^{\mathrm{m}} 02^{\mathrm{s}} .259,+30^{\circ} 34^{\prime} 19^{\prime \prime}\right.$. 18$)$

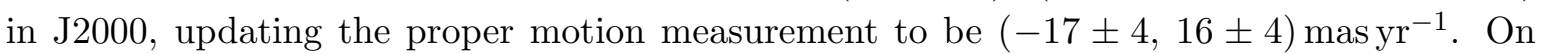
the other hand, the ${ }^{12} \mathrm{CO} J=2-1$ emission peak position rotates counterclockwise with the channel velocity, staying on the northwest side $(<\Delta \mathrm{RA}\rangle=-70 \pm 60$ mas; $<\Delta$ Dec $>=$ $40 \pm 70$ mas) of the dust continuum emission center. The positional shift of molecular line emission peak in the proper motion direction is noticeable.

- Continuum emission at $226.2 \mathrm{GHz}$ from the SMA observations best fits to a two-component model consisting of an unresolved source (of flux $26.4 \mathrm{mJy}$ ) and an extended component with a size $\lesssim 4^{\prime \prime}(34.5 \mathrm{mJy})$. The unresolved continuum source is also observed at $350.6 \mathrm{GHz}$ and has a spectral index $\sim-2$, consistent with thermal blackbody emission from a radio photosphere with a diameter of $8 \mathrm{AU}$ and the temperature of $2000 \mathrm{~K}$.

- The systemic LSR velocity of $-2 \mathrm{~km} \mathrm{~s}^{-1}$ is defined as the middle velocity between the double peak spectral profile of the ${ }^{13} \mathrm{CO} J=2-1$ line in the innermost $1^{\prime \prime}$ region. With respect to the systemic velocity, the peaks are located at $\pm 16 \mathrm{~km} \mathrm{~s}^{-1}$ and its brightness temperature reaches zero at $\sim \pm 18 \mathrm{~km} \mathrm{~s}^{-1}$. The outer envelope has an additional $-10 \mathrm{~km} \mathrm{~s}^{-1}$ component, brighter than the $-16 \mathrm{~km} \mathrm{~s}^{-1}$ peak.

- The ${ }^{12} \mathrm{CO} J=2-1$ emission spectral profile varies with integration radius; a red-skewed double peak profile (with a small blue-end bump) over $r \leq 1^{\prime \prime}$, a nearly flat-topped profile over $r \leq 5^{\prime \prime}$, and a parabolic shaped profile from the single dish observations with $\mathrm{a} \sim 33^{\prime \prime}$-sized beam. It shows the optical depth change with radius; i.e., the innermost envelope is relatively optically thin and a significant fraction of $5^{\prime \prime}$ region becomes optically thick.

- The spatially-averaged radial and spectral profiles of ${ }^{12} \mathrm{CO} J=2-1$ in all length scales are reasonably well reproduced by a simple spherical radiative transfer model. However, the fixed fractional abundance model fails in matching the overall radial distribution of the observed

${ }^{13} \mathrm{CO} J=2-1$ emission. This may suggest that the ${ }^{12} \mathrm{CO} /{ }^{13} \mathrm{CO}$ abundance ratio varies in the CSE of CIT 6 from $\sim 30$ in the outer envelope to $\sim 50$ in the inner envelope.

- The high resolution ${ }^{12} \mathrm{CO} J=2-1$ map (from the SMA) reveals a spiral-shell pattern appearing as a $20 \%$ fluctuation of the average profile, due to the suggested binary orbital motion. This pattern is undetected in the ${ }^{13} \mathrm{CO} J=2-1$ emission likely due to sensitivity, as $20 \%$ of the observed ${ }^{13} \mathrm{CO}$ maximum brightness corresponds to $\sim 1 \sigma$ only. 
- The previously observed, arc-shaped pattern in the $\mathrm{HC}_{3} \mathrm{~N} J=4-3$ emission map turns out to be an incomplete form of the spiral-shell pattern appearing in the ${ }^{12} \mathrm{CO} J=2-1$ map. The $\mathrm{HC}_{3} \mathrm{~N}$ segments (broken to the west side and central $3^{\prime \prime}$ region) are connected by the ${ }^{12} \mathrm{CO}$ pattern. The $\mathrm{HC}_{3} \mathrm{~N} J=4-3$ line emission likely traces the shocked arm region with high arm-interarm contrasts.

- A bipolar outflow of CIT 6 is found for the first time in the ${ }^{12} \mathrm{CO} J=2-1$ channel map. The blue component (at $-19 \mathrm{~km} \mathrm{~s}^{-1}$ ) is separated from the AGB envelope by a gap at $-16 \mathrm{~km} \mathrm{~s}^{-1}$, clearly seen in the P-V diagrams. The red counterpart (at $19 \mathrm{~km} \mathrm{~s}^{-1}$ ) is smaller than the blue component of the bipolar outflow, and points to a nearly perpendicular direction.

- In the zoomed-in channel map and spectrum, the $\sim 10 \mathrm{~km} \mathrm{~s}^{-1}$ channels exhibit the strongest emission, indicating that the spectral asymmetry of the ${ }^{12} \mathrm{CO} J=2-1$ emission originates from the innermost region. In contrast, the source of the red-skewed profile of $\mathrm{HC}_{3} \mathrm{~N} J=4-3$ can be found from the broadly distributed outer shells at $v_{z} \gtrsim 10 \mathrm{~km} \mathrm{~s}^{-1}$.

- An eccentric binary orbit is hinted in the ${ }^{12} \mathrm{CO} J=2-1$ map by a double spiral feature at the systemic velocity and by the lack of interarm emission to the west, indicating the location of orbit periastron. The pattern spacing changes from $\sim 2$ ". 5 between the outermost arms beyond $\sim 7^{\prime \prime}$ to $\sim 1^{\prime \prime} .5$ between the inner arms, characterizing an either reduced orbital period or slower wind flow.

\section{CONCLUSION}

Our ${ }^{12} \mathrm{CO} J=2-1$ maps reveal a circumstellar spiral-shell pattern and a nascent bipolar outflow, providing an observational support for the binary system scenario for CIT 6 . In addition, the data reveal an inner double spiral and interarm gaps in one direction as possible evidences for an eccentric binary. The eccentric orbital motion of a wide binary could facilitate the formation of a necessary accretion disk surrounding the companion to trigger a bipolar outflow emerging in the AGB-pPN transition.

We thank all SMA and SMT staffs for supporting the observations, and the anonymous referee for helpful suggestions to improve this paper. H.K. acknowledges support through EACOA Fellowship from the East Asia Core Observatories Association, which consists of the National Astronomical Observatories of China, the National Astronomical Observatory of Japan, the Korea Astronomy and Space Science Institute, and the Academia Sinica Institute of Astronomy and Astrophysics. F.K. is supported by the Ministry of Science and Technology (MoST) of Taiwan, under grant numbers MOST103-2112-M-001-033- and MOST104-2628-M-001-004-MY3. 


\section{REFERENCES}

Balick, B., \& Frank, A. 2002, ARA\&A, 40, 439

Beichman, C. A., Neugebauer, G., Habing, H. J., Clegg, P. E., \& Chester, T. J. 1988, Infrared astronomical satellite (IRAS) catalogs and atlases, Explanatory supplement, 1

Carlstrom, J. E., Welch, W. J., Goldsmith, P. F., \& Lis, D. C. 1990, AJ, 100, 213

Castro-Carrizo, A., Quintana-Lacaci, G., Neri, R., et al. 2010, A\&A, 523, A59

Cernicharo, J., Marcelino, N., Agúndez, M., \& Guélin, M. 2015, A\&A, 575, A91

Chau, W., Zhang, Y., Nakashima, J.-i., Deguchi, S., \& Kwok, S. 2012, ApJ, 760, 66

Claussen, M. J., Kleinmann, S. G., Joyce, R. R., \& Jura, M. 1987, ApJS, 65, 385

Claussen, M. J., Sjouwerman, L. O., Rupen, M. P., et al. 2011, ApJ, 739, L5

Cohen, M. 1979, MNRAS, 186, 837

Cohen, M., \& Hitchon, K. 1996, AJ, 111, 962

Corradi, R. L. M., \& Schwarz, H. E. 1995, A\&A, 293, 871

Decin, L., Richards, A. M. S., Neufeld, D., et al. 2015, A\&A, 574, A5

Dinh-V.-Trung, \& Lim, J. 2009, ApJ, 701, 292

Groenewegen, M. A. T., Waelkens, C., Barlow, M. J., et al. 2011, A\&A, 526, A162

Gurwell, M. A., Peck, A. B., Hostler, S. R., Darrah, M. R., \& Katz, C. A. 2007, in ASP Conf. Ser. 375, From Z-Machines to ALMA: (Sub)Millimeter Spectroscopy of Galaxies, ed. A. J. Baker, J. Glenn, A. I. Harris, J. G. Mangum, \& M. S. Yun (San Francisco, CA: ASP), 234

He, J. H. 2007, A\&A, 467, 1081

Höfner, S. 2007, Why Galaxies Care About AGB Stars: Their Importance as Actors and Probes, 378,145

Huarte-Espinosa, M., Carroll-Nellenback, J., Nordhaus, J., Frank, A., \& Blackman, E. G. 2013, MNRAS, 1448

Huggins, P. J., Mauron, N., \& Wirth, E. A. 2009, MNRAS, 396, 1805

Hurley, J. R., Pols, O. R., \& Tout, C. A. 2000, MNRAS, 315, 543

Kim, H., Hsieh, I.-T., Liu, S.-Y., \& Taam, R. E. 2013, ApJ, 776, 86

Kim, H., \& Taam, R. E. 2012a, ApJ, 744, 136 
Kim, H., \& Taam, R. E. 2012b, ApJ, 759, 59

Kim, H., \& Taam, R. E. 2012c, ApJ, 759, L22

Knapp, G. R., Kuiper, T. B. H., \& Zuckerman, B. 1979, ApJ, 233, 140

Koda, J., Sawada, T., Wright, M. C. H., et al. 2011, ApJS, 193, 19

Lagadec, E., Mékarnia, D., de Freitas Pacheco, J. A., \& Dougados, C. 2005, A\&A, 433, 553

Lindqvist, M., Schöier, F. L., Lucas, R., \& Olofsson, H. 2000, A\&A, 361, 1036

Loup, C., Forveille, T., Omont, A., \& Paul, J. F. 1993, A\&AS, 99, 291

Maercker, M., Mohamed, S., Vlemmings, W. H. T., et al. 2012, Nature, 490, 232

Mamon, G. A., Glassgold, A. E., \& Huggins, P. J. 1988, ApJ, 328, 797

Marshall, C. R., Leahy, D. A., \& Kwok, S. 1992, PASP, 104, 397

Mastrodemos, N., \& Morris, M. 1999, ApJ, 523, 357

Mauron, N., \& Huggins, P. J. 1999, A\&A, 349, 203

Mauron, N., \& Huggins, P. J. 2006, A\&A, 452, 257

Mauron, N., Huggins, P. J., \& Cheung, C.-L. 2013, A\&A, 551, A110

Mayer, A., Jorissen, A., Kerschbaum, F., et al. 2011, A\&A, 531, L4

Menten, K. M., Reid, M. J., Kamiński, T., \& Claussen, M. J. 2012, A\&A, 543, AA73

Milam, S. N., Woolf, N. J., \& Ziurys, L. M. 2009, ApJ, 690, 837

Mohamed, S., \& Podsiadlowski, P. 2012, Baltic Astronomy, 21, 88

Monnier, J. D., Tuthill, P. G., \& Danchi, W. C. 2000, ApJ, 545, 957

Morris, M. 1975, ApJ, 197, 603

Morris, M., Lucas, R., \& Omont, A. 1985, A\&A, 142, 107

Morris, M., Sahai, R., Matthews, K., et al. 2006, in IAU Symp. 234, Planetary Nebulae in our Galaxy and Beyond, ed. M. J. Barlow \& R. H. Méndez (Cambridge: Cambridge Univ. Press), 469

Neri, R., Kahane, C., Lucas, R., Bujarrabal, V., \& Loup, C. 1998, A\&AS, 130, 1

Olofsson, H., Eriksson, K., Gustafsson, B., \& Carlstrom, U. 1993, ApJS, 87, 267 
Ramstedt, S., Mohamed, S., Vlemmings, W. H. T., et al. 2014, A\&A, 570, L14

Reid, M. J., \& Menten, K. M. 1997, ApJ, 476, 327

Röser, S., Schilbach, E., Schwan, H., et al. 2008, A\&A, 488, 401

Sahai, R., Morris, M., Sánchez Contreras, C., \& Claussen, M. 2007, AJ, 134, 2200

Sahai, R., Trauger, J. T., Watson, A. M., et al. 1998, ApJ, 493, 301 /

Sahai, R., \& Mack-Crane, G. P. 2014, AJ, 148, 74

Schmidt, G. D., Hines, D. C., \& Swift, S. 2002, ApJ, 576, 429

Schöier, F. L., \& Olofsson, H. 2001, A\&A, 368, 969

Schöier, F. L., van der Tak, F. F. S., van Dishoeck, E. F., \& Black, J. H. 2005, A\&A, 432, 369

Soker, N. 1994, MNRAS, 270, 774

Theuns, T., \& Jorissen, A. 1993, MNRAS, 265, 946

Wood, P. R. 1979, ApJ, 227, 220

Zhang, Y., Kwok, S., \& Dinh-V-Trung 2009, ApJ, 691, 1660

This preprint was prepared with the AAS LATEX macros v5.2.

Table 1. SMA observation summary

\begin{tabular}{cccc}
\hline \hline Date & Configuration & Integration (hour) & $T_{\text {sys }}(\mathrm{K})$ \\
\hline $2013-01-08$ & extended & 4.4 & $70-140$ \\
$2013-02-06$ & very extended & 3.6 & $70-140$ \\
$2013-12-12$ & compact & 3.4 & $90-160$ \\
$2014-02-09$ & subcompact & 0.8 & $80-150$ \\
$2014-03-11$ & subcompact & 3.8 & $90-140$ \\
\hline
\end{tabular}


Table 2. SMT grid-mapping observation summary

\begin{tabular}{cccccc}
\hline \hline Molecule & Date & Grid & Spacing $\left({ }^{\prime \prime}\right)$ & Integration $(\min )$ & $T_{\text {sys }}(\mathrm{K})$ \\
\hline${ }^{12} \mathrm{CO}(2-1)$ & $2015-01-07$ & $9 \times 9$ & 15 & $3 \times 81$ & $160-190$ \\
\hline${ }^{13} \mathrm{CO}(2-1)$ & $2015-01-07$ & $9 \times 9$ & 15 & $3 \times 81$ & $170-210$ \\
& $2015-01-17$ & $7 \times 7$ & 15 & $3 \times 49$ & $180-280$ \\
\hline
\end{tabular}

Table 3. Continuum $u v$-fitting results

\begin{tabular}{cccccccc}
\hline \hline $\begin{array}{c}\nu \\
(\mathrm{GHz})\end{array}$ & $\begin{array}{c}u v \text { coverage } \\
(\mathrm{k} \lambda)\end{array}$ & Model & $\begin{array}{c}F_{\nu} \\
(\mathrm{mJy})\end{array}$ & $\begin{array}{c}\text { Major axis } \\
\left({ }^{\prime \prime}\right)\end{array}$ & $\begin{array}{c}\text { Minor axis } \\
\left({ }^{\prime \prime}\right)\end{array}$ & $\begin{array}{c}\Delta \mathrm{RA}^{\mathrm{a}} \\
(\mathrm{mas})\end{array}$ & $\begin{array}{c}\Delta \mathrm{Dec}^{\mathrm{a}} \\
(\mathrm{mas})\end{array}$ \\
\hline 226.2 & $4-384$ & $\begin{array}{c}\text { Point }+ \\
\text { Gaussian }\end{array}$ & $\begin{array}{c}26.4 \pm 0.5 \\
34.5 \pm 1.2\end{array}$ & $3.7 \pm 0.2$ & $3.0 \pm 0.2$ & $\begin{array}{c}0 \pm 5 \\
20 \pm 70\end{array}$ & $\begin{array}{c}0 \pm 4 \\
50 \pm 63\end{array}$ \\
\hline \hline 350.6 & $42-596$ & Point & $77.4 \pm 2.6$ & & & $-20 \pm 6$ & $-10 \pm 6$ \\
\hline
\end{tabular}

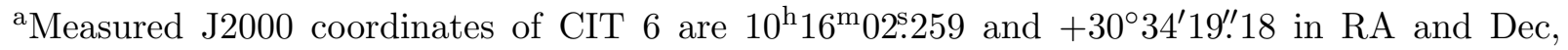
respectively. 


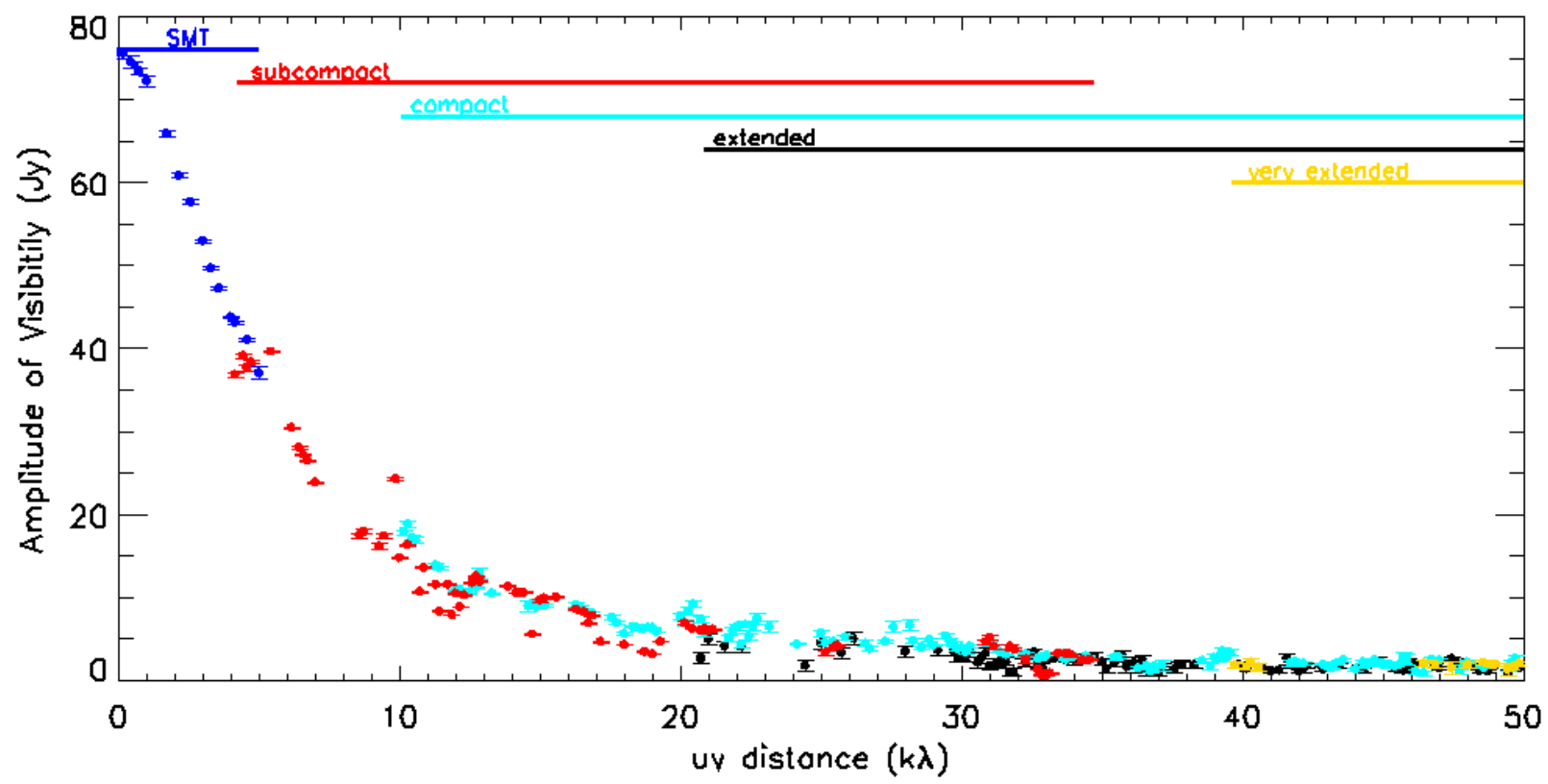

Fig. 1. - Visibility amplitude of ${ }^{12} \mathrm{CO} J=2-1$ line emission from CIT 6 (integrated over $40 \mathrm{~km} \mathrm{~s}^{-1}$ ) along the $u v$ distance of the SMT (blue; 2015 Jan. 07 and 17) and SMA's subcompact (red; 2014 Feb. 09 and Mar. 11), compact (cyan; 2013 Dec. 12), extended (black; 2013 Jan. 08) and very extended (yellow; 2013 Feb. 06) configurations. The visibility data are shown with 350 bins in the $u v$ distance up to $50 \mathrm{k} \lambda$. The full $u v$ distance coverage is up to $400 \mathrm{k} \lambda$. 


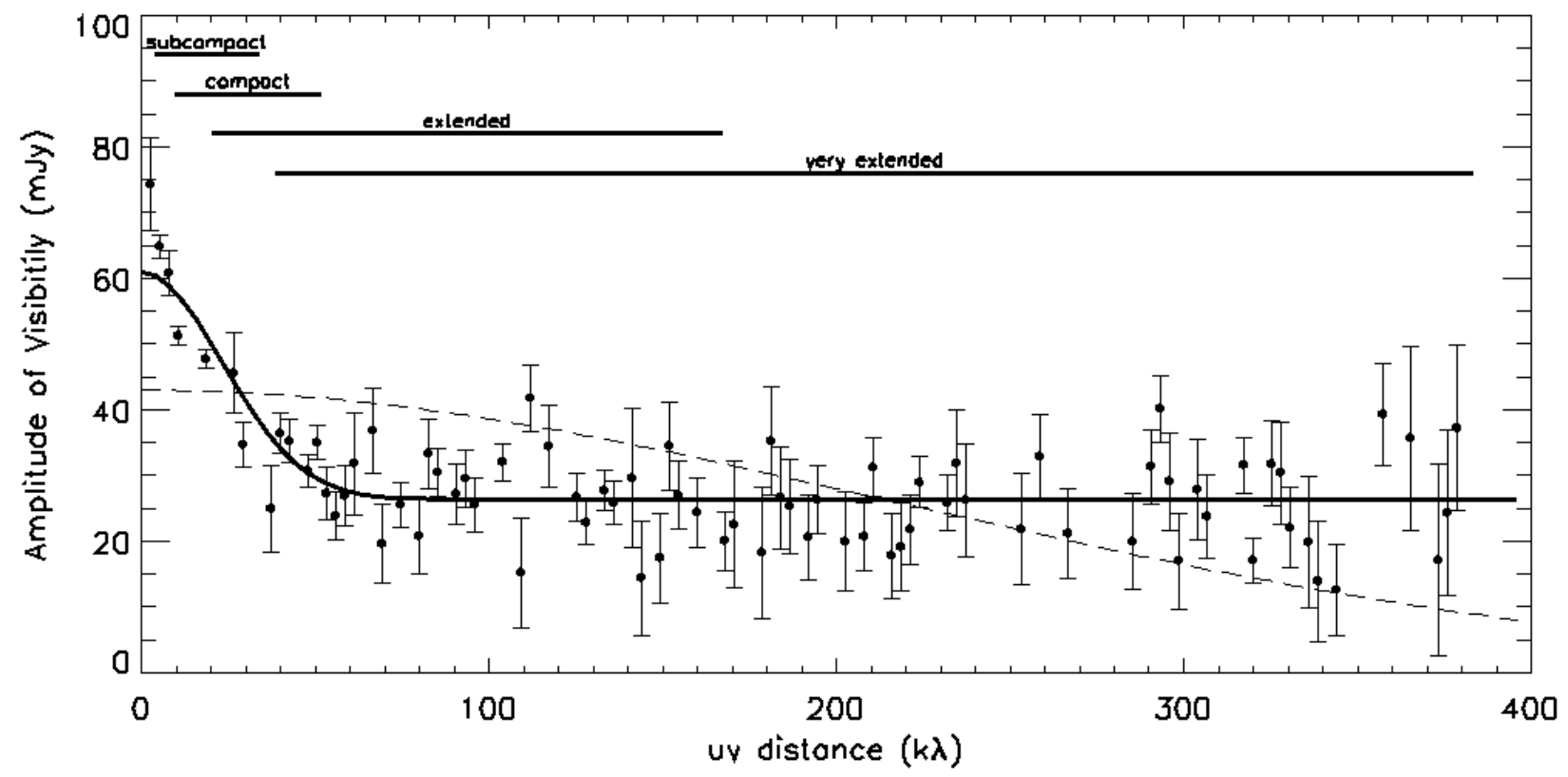

Fig. 2.- Visibility amplitude at $226.2 \mathrm{GHz}$ continuum along the $u v$ distance of the SMA, averaged per $2.65 \mathrm{k} \lambda$-width bin. Solid curve represents our two-component (one point and one Gaussian) model (see Table 3). For comparison, a single Gaussian model is overlaid by a dashed curve.
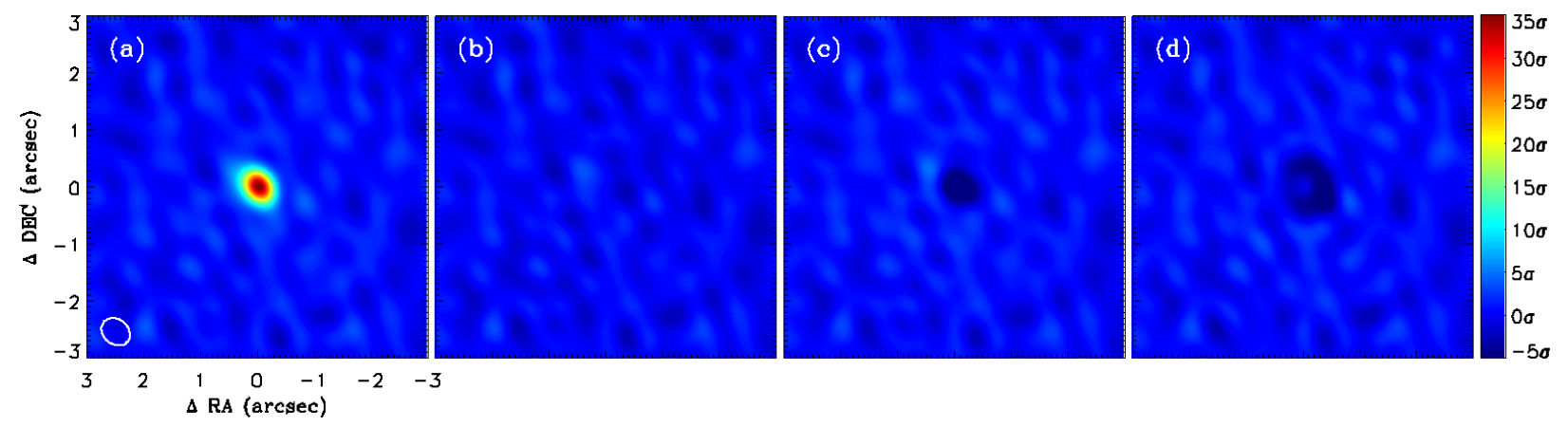

Fig. 3.- (a) Map of the continuum emission at 226.2 GHz taken with the SMA all configurations. Residuals after subtracting (b) two-component (point source + Gaussian) model, (c) one point source model, and (d) one Gaussian model. The synthesized beam size is indicated by a white contour in (a). The colorbar is labeled in a linear scale in units of the noise level $\sigma=0.75 \mathrm{mJy} \mathrm{beam}^{-1}$. 


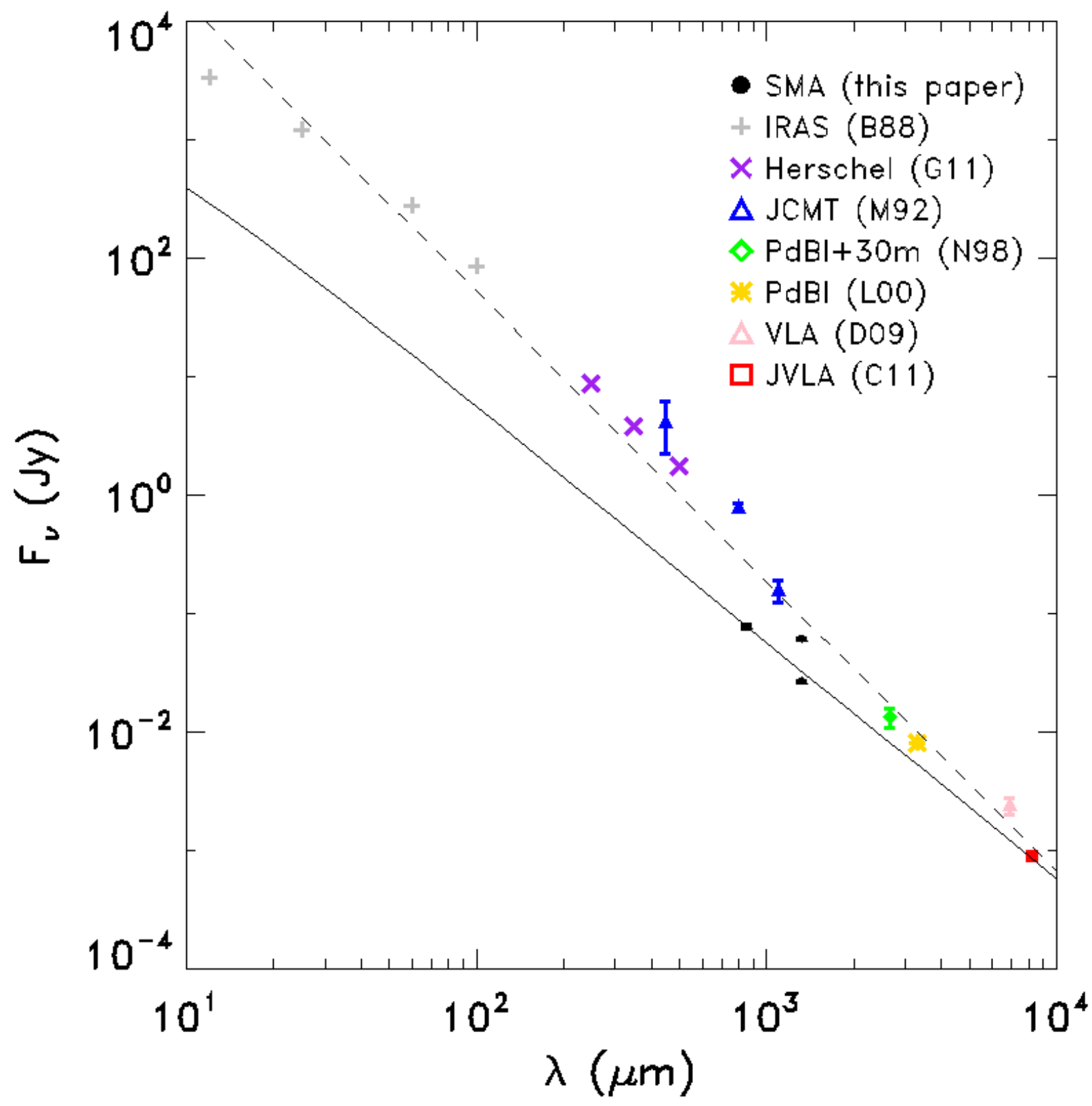

Fig. 4.- Spectral energy distribution of CIT 6 from infrared to centimeter wavelengths $(\sim 10 \mu \mathrm{m}$ to $\sim 1 \mathrm{~cm})$. The SMA flux at $226.2 \mathrm{GHz}(1.3 \mathrm{~mm})$ and $350.6 \mathrm{GHz}(0.8 \mathrm{~mm})$ are measured via visibility fits. Solid line represents thermal black body radiation curve from the suggested radio photosphere of CIT 6 with a temperature of $2000 \mathrm{~K}$ and a diameter of $8 \mathrm{AU}$ (20 mas). Dashed line shows a linear fit to the accumulated data from the literature, resulting in a spectral index of -2.4 . The references B88, G11, M92, N98, L00, D09, and C11 represent Beichman et al. (1988), Groenewegen et al. (2011), Marshall et al. (1992), Neri et al. (1998), Lindqvist et al. (2000), Dinh-V.-Trung \& $\operatorname{Lim}(2009)$, and Claussen et al. (2011), respectively. 

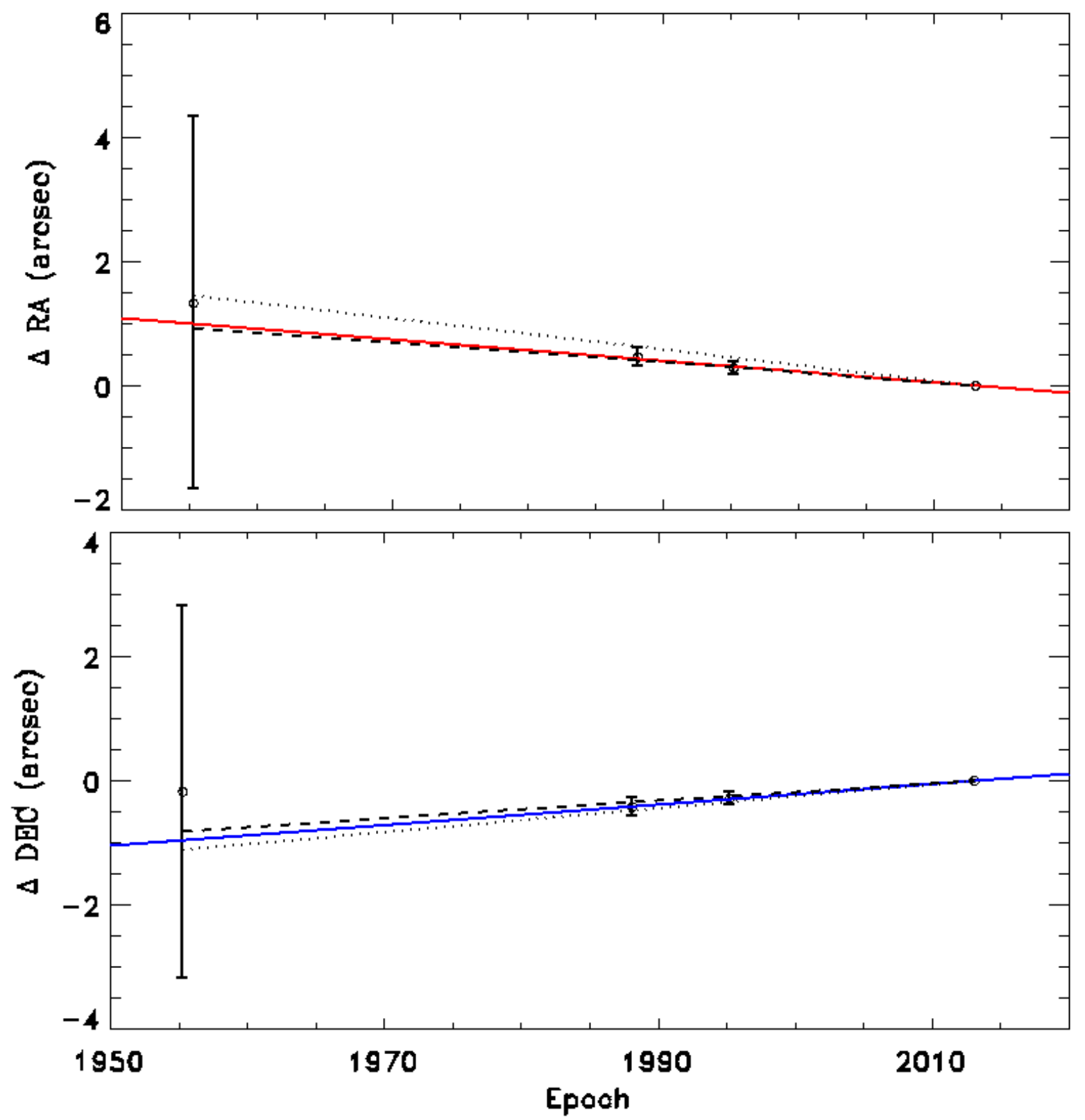

Fig. 5.- Proper motion of CIT 6 based on the continuum emission at $226.2 \mathrm{GHz}$ from the SMA (epoch 2013.1), compared with the continuum emission at $90.7 \mathrm{GHz}$ (in epoch 1995.2, Lindqvist et al. 2000), the HCN maser (Carlstrom et al. 1990, in epoch 1988.11), and the optical image (Claussen et al. 1987, in epoch 1955.29). The colored solid lines present our proper motion measurement by linear fitting of the four data points, and the dashed and dotted lines indicate earlier measurements by Monnier et al. (2000) and Röser et al. (2008), respectively. 

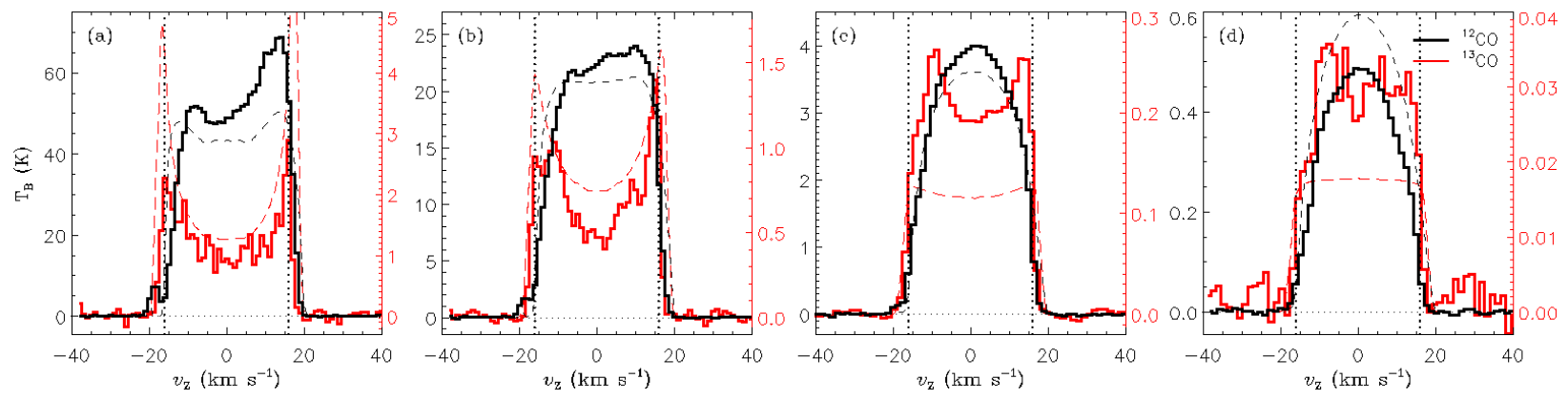

Fig. 6. - Spectra of the observed ${ }^{12} \mathrm{CO} J=2-1$ (black solid line) and ${ }^{13} \mathrm{CO} J=2-1$ (red solid) emission from (a) the central region $\left(r \leq 1^{\prime \prime}\right)$ of the SMA-SMT combined images, (b) the $r \leq 5^{\prime \prime}$ region, (c) the single pointing with the SMT (beam size $\sim 33^{\prime \prime}$ ), and (d) the entire region of the SMT grid-map $(\sim 2.5 \times 2.5)$ in units of brightness temperature. One-dimensional radiative transfer model spectra with the ${ }^{12} \mathrm{CO} /{ }^{13} \mathrm{CO}$ abundance ratio of 50 is presented by dashed lines in the same color-codes. The vertical axes of ${ }^{12} \mathrm{CO}$ line spectra are scaled by 15 from the ${ }^{13} \mathrm{CO}$ axes. Vertical dotted lines mark $v_{z}= \pm 16 \mathrm{~km} \mathrm{~s}^{-1}$, where $v_{z}=v_{\mathrm{LSR}}-v_{\mathrm{sys}}$ and $v_{\mathrm{sys}}=-2 \mathrm{~km} \mathrm{~s}^{-1}$.
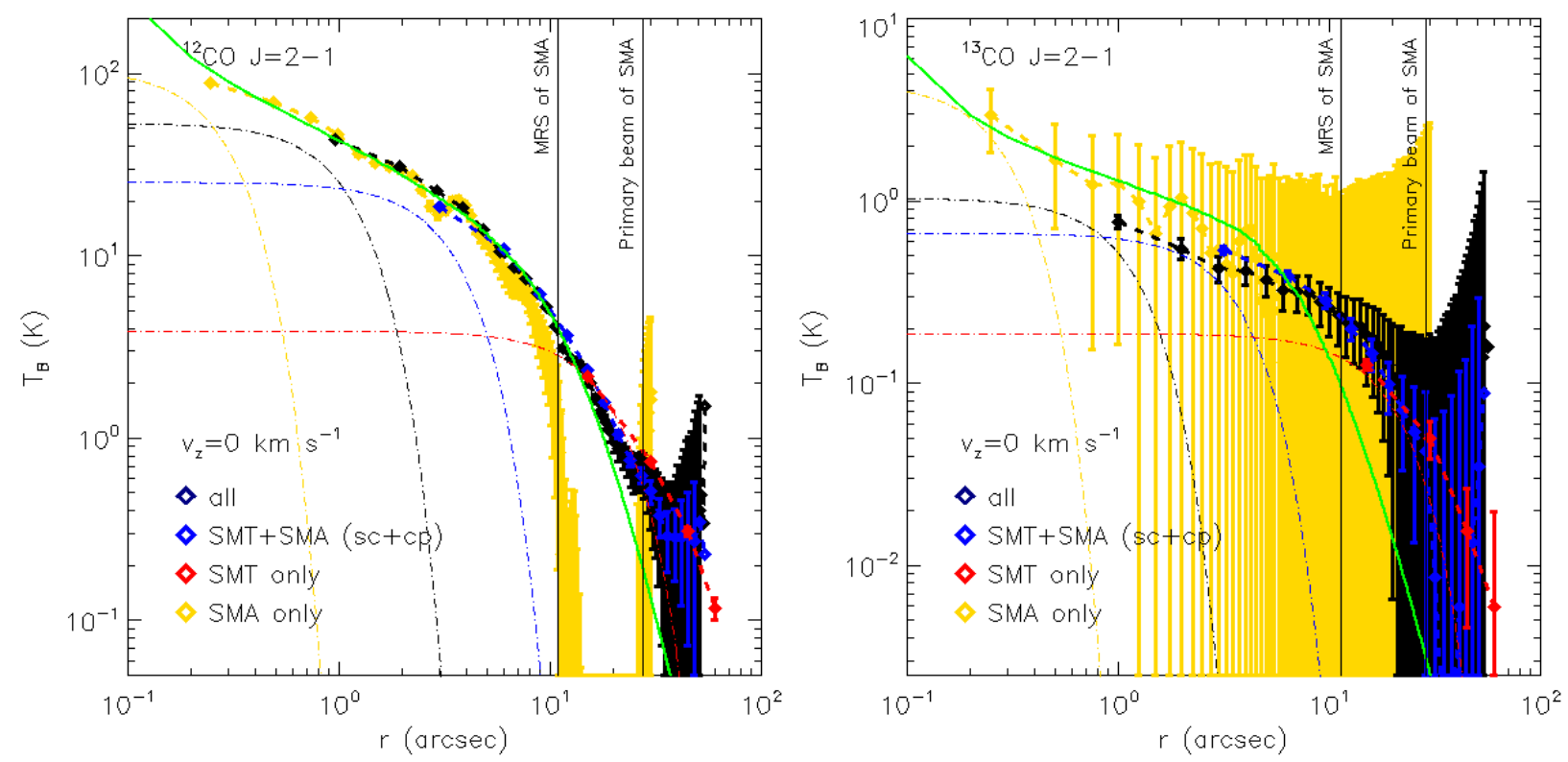

Fig. 7. - Radial profiles of ${ }^{12} \mathrm{CO} J=2-1$ (left panel) and ${ }^{13} \mathrm{CO} J=2-1$ (right panel) at the systemic velocity (width of $5 \mathrm{~km} \mathrm{~s}^{-1}$ ) in different configurations of the array. Our radiative transfer model is overlaid by green curves (see text for detail). Dash-dotted lines represent the restored Gaussian beams in the same color codes for the data points. The maximum recoverable scale and primary beam of the SMA are marked in each panel. 


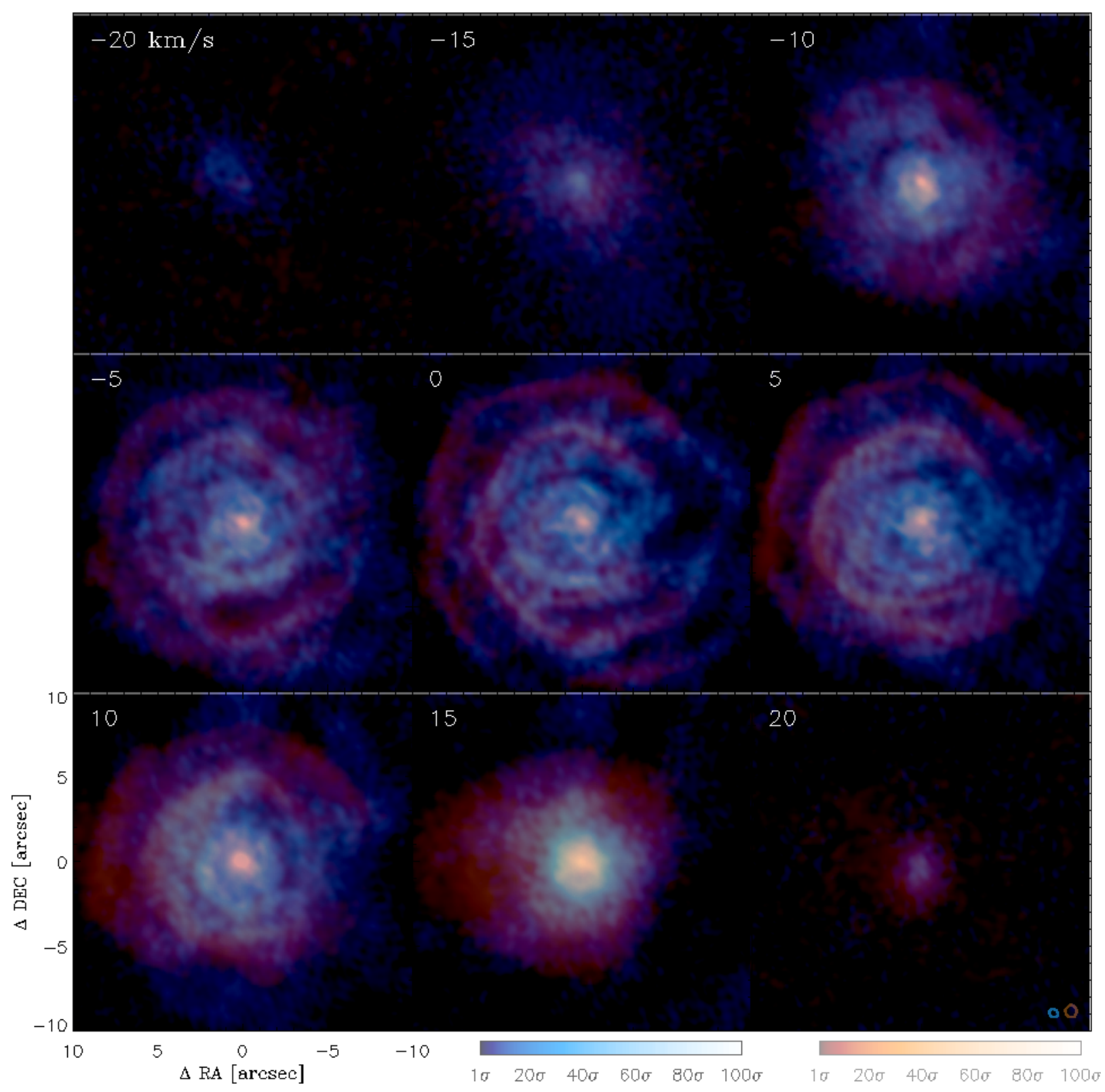

Fig. 8. - Channel map of the ${ }^{12} \mathrm{CO} J=2-1$ emission (blue) from the SMA observation, overlaid with that of $\mathrm{HC}_{3} \mathrm{~N} J=4-3$ emission (red; Claussen et al. 2011). Each panel displays the channel image averaged over $5 \mathrm{~km} \mathrm{~s}^{-1}$, and is labeled by $v_{z}=v_{\mathrm{LSR}}-v_{\mathrm{sys}}$ (that is, the channel velocity with respect to the systemic velocity $v_{\mathrm{sys}}=-2 \mathrm{~km} \mathrm{~s}^{-1}$ ). The image center is set to the position of the peak of continuum emission at $226.2 \mathrm{GHz}$. The synthesized beam sizes of the ${ }^{12} \mathrm{CO}$ and $\mathrm{HC}_{3} \mathrm{~N}$ lines are denoted at the bottom right corner in the corresponding colors. Colorbars are marked in units of the noise levels for a velocity width of $5 \mathrm{~km} \mathrm{~s}^{-1} ; \sigma=12 \mathrm{mJy}_{\text {beam }}{ }^{-1}(1.1 \mathrm{~K})$ for the ${ }^{12} \mathrm{CO}$ map, and $\sigma=0.24$ mJy beam $^{-1}(0.46 \mathrm{~K})$ for the $\mathrm{HC}_{3} \mathrm{~N}$ map. 


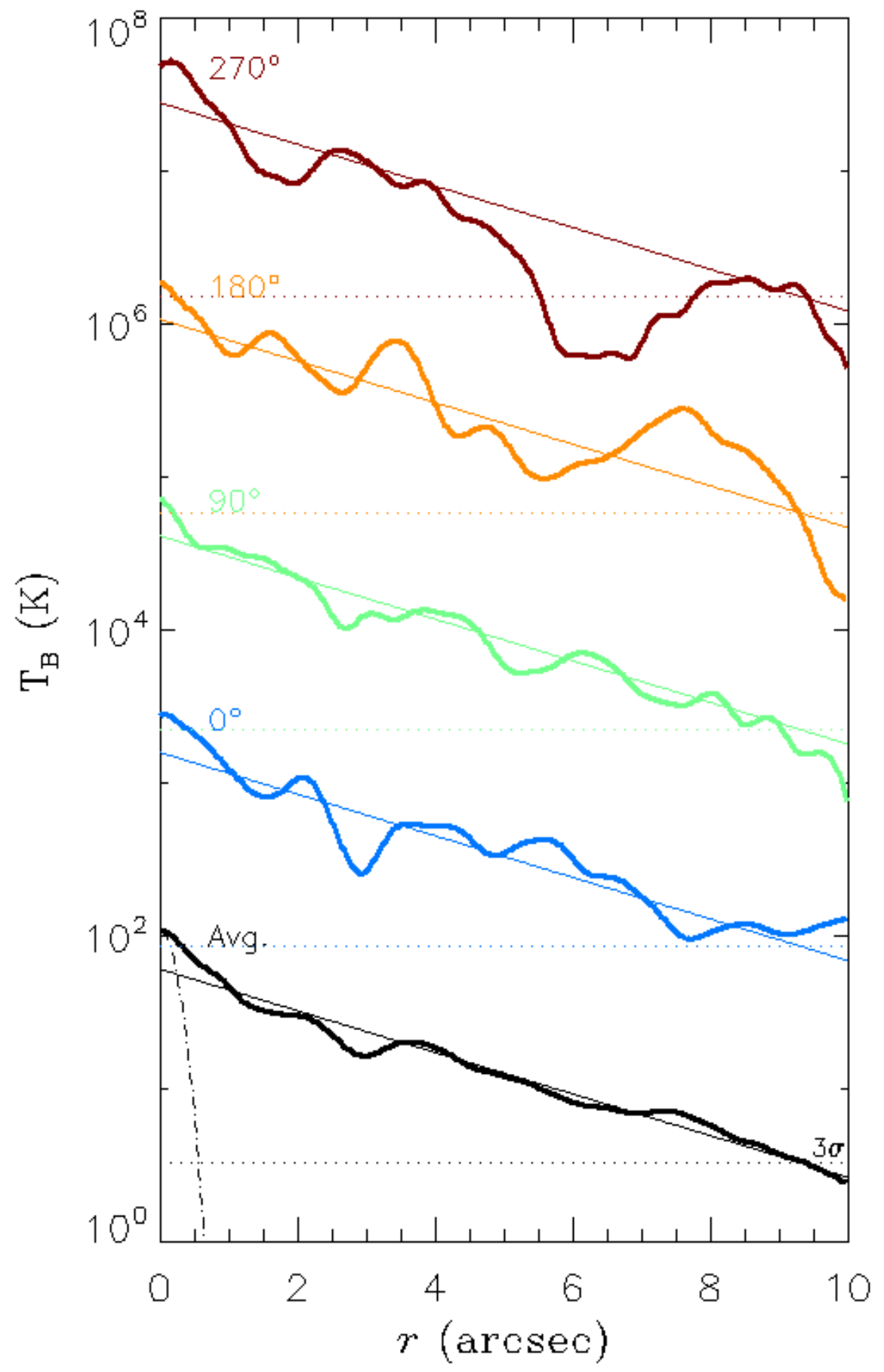

Fig. 9.- Radial profiles of ${ }^{12} \mathrm{CO} J=2-1$ at the systemic velocity (width of $5 \mathrm{~km} \mathrm{~s}^{-1}$ ). Bottom black curve presents the azimuthally averaged profile over $360^{\circ}$, overlaid by a thin line representing a linear fit (slope of -0.31) in the log-linear scale (i.e., an exponential function). Dotted and dashdotted lines indicate the $3 \sigma$ level and the synthesized Gaussian beam, respectively. The upper four curves represent the profiles along the noted PA averaged over 20 degree-width sectors. They are vertically shifted (by 3.3 in the logarithmic scale) together with the thin solid and dotted lines, which denote analogous quantities (averaged fit and $3 \sigma$ level, respectively) as for the black curves but for the different PAs. 

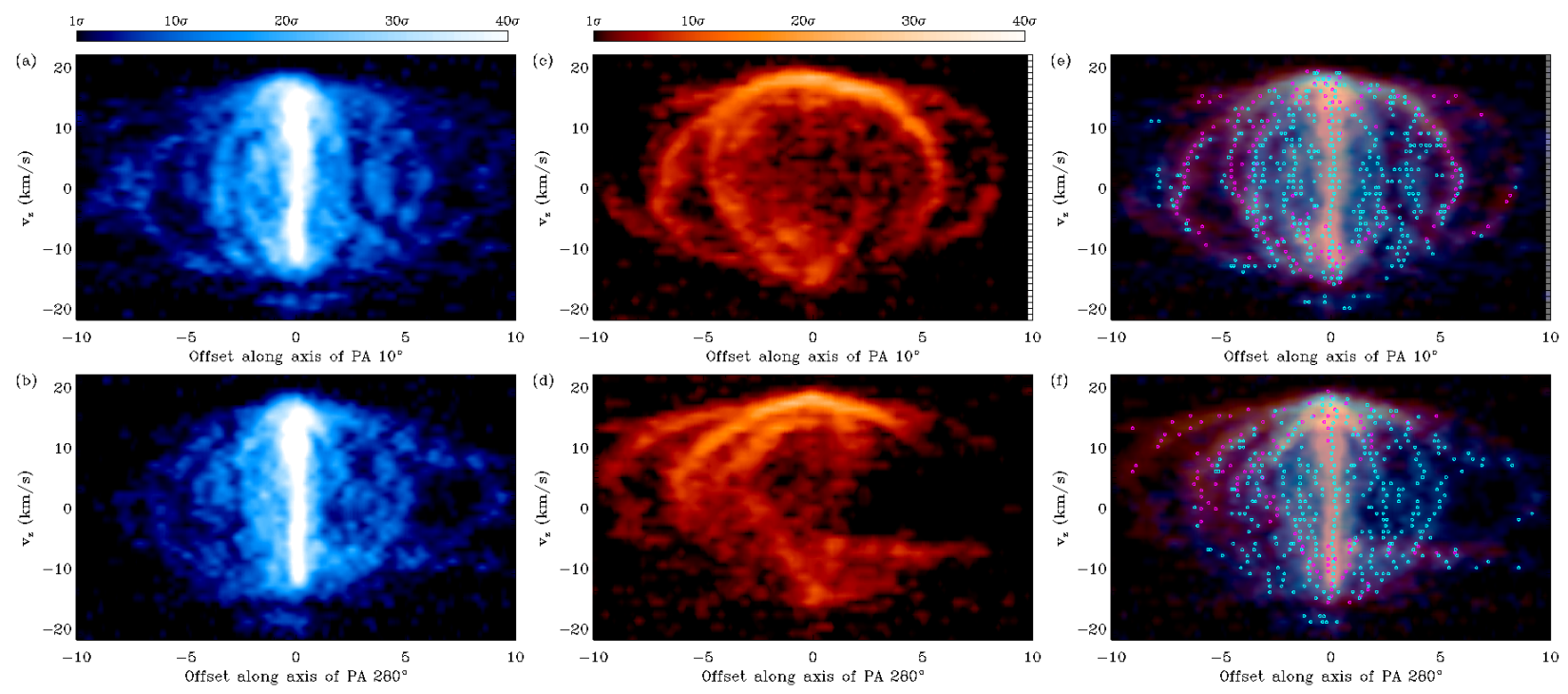

Fig. 10. - Position-velocity diagrams of the ${ }^{12} \mathrm{CO} J=2-1$ (blue) and $\mathrm{HC}_{3} \mathrm{~N} J=4-3$ (red) for PAs of $10^{\circ}$ (upper panels) and $280^{\circ}$ (lower panels). The negative offset means the opposite direction, i.e., PA of $190^{\circ}$ (upper) and $100^{\circ}$ (lower). The velocity interval is $1 \mathrm{~km} \mathrm{~s}^{-1}$, and is denoted with respect to the systemic velocity. The open circles in (e)-(f) refer the local peak positions selected with the criteria of $10 \sigma$ for ${ }^{12} \mathrm{CO}\left(\sigma=24 \mathrm{mJy}_{\text {beam }}{ }^{-1}\right)$ and $5 \sigma$ for $\mathrm{HC}_{3} \mathrm{~N}\left(\sigma=0.5 \mathrm{mJybeam}^{-1}\right)$. 


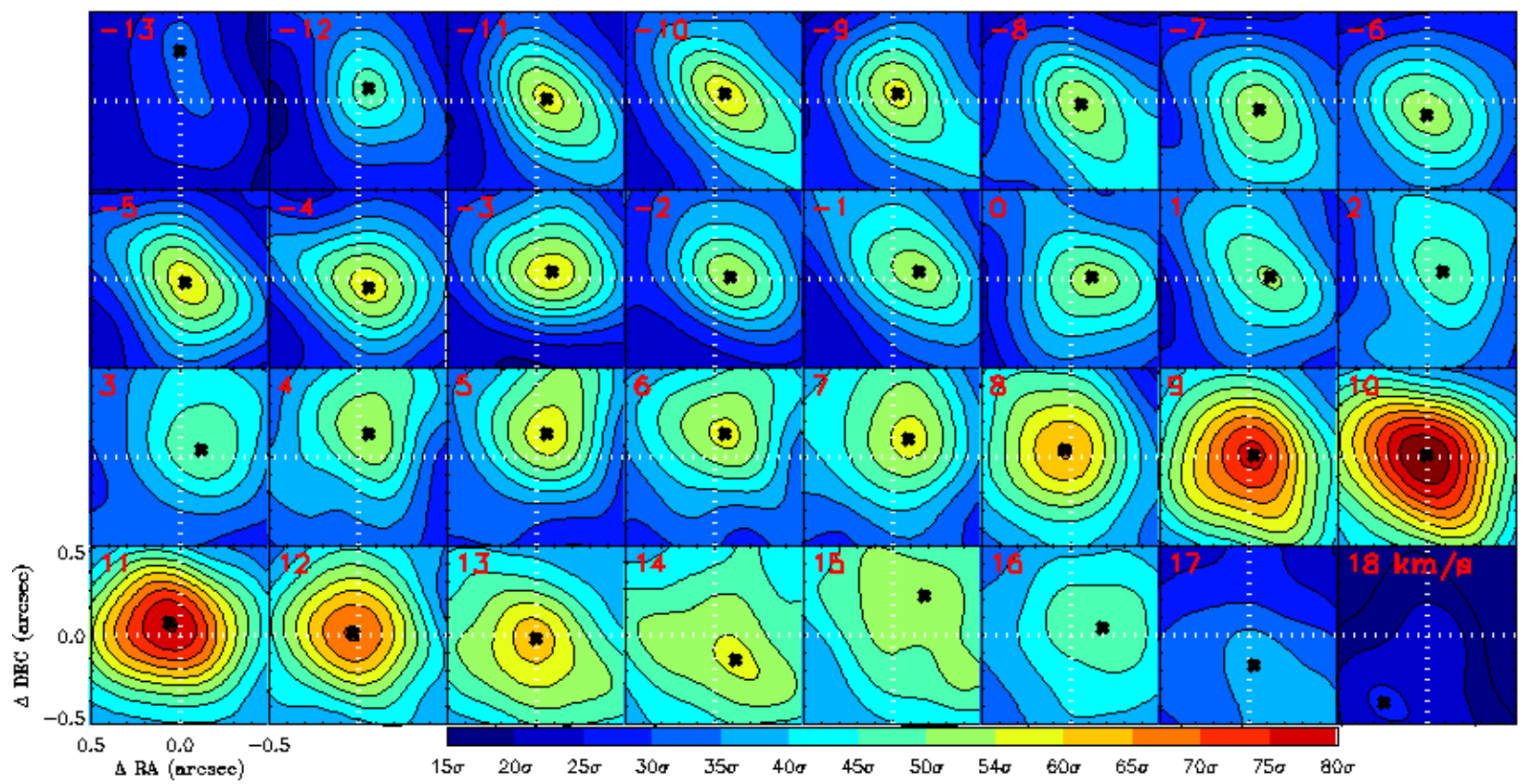

Fig. 11. - Channel map of ${ }^{12} \mathrm{CO} J=2-1$ in the central region of CIT 6, taken with the SMA. The emission peak position of each channel is marked by a cross symbol. The number at the top left corner of each panel represents the center velocity of the channel with respect to the systemic velocity. The colorbar and contour levels are from $15 \sigma$ to $80 \sigma$ with a step of $5 \sigma$, where the noise level $\sigma=24$ mJy beam $^{-1}(2.2 \mathrm{~K})$. Continuum peak position is indicated by the intersection of dotted lines. 

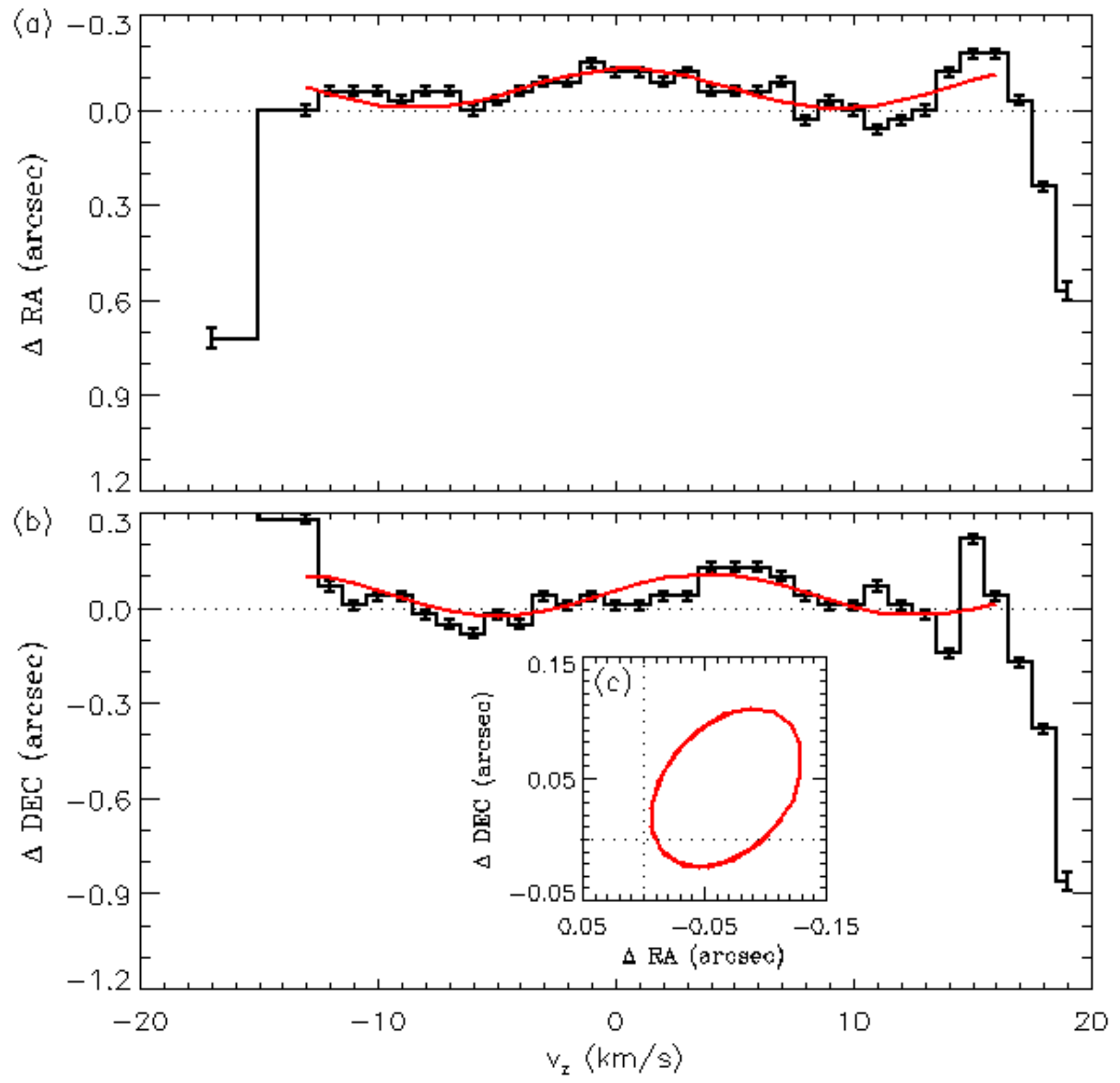

Fig. 12.- Peak position of the ${ }^{12} \mathrm{CO} J=2-1$ line emission in each channel along (a) RA and (b) Dec directions separately, with respect to the coordinate center defined by the peak of the continuum emission at $226.2 \mathrm{GHz}$. The red lines show the fitting results of the offsets with a functional form of $f=a_{0}+a_{1} \sin \left(2 \pi\left(v_{z}-a_{3}\right) / a_{2}\right)$. The resulting fit constants are $\left(a_{0}, a_{1}, a_{2}, a_{3}\right)=$ $(-0.07,0.06,17.7,4.89)$ for RA and $(0.04,0.07,17.7,-0.58)$ for Dec. The vertical bars represent the position measurement uncertainties defined by the larger values between the synthesized beam size divided by the signal-to-noise ratio and the pixel size of the channel map. (c) The position curve in $\triangle \mathrm{RA}$ and $\triangle \mathrm{DEC}$ are based upon the fits from (a) and (b). Dotted lines are inserted to show the continuum peak position. 


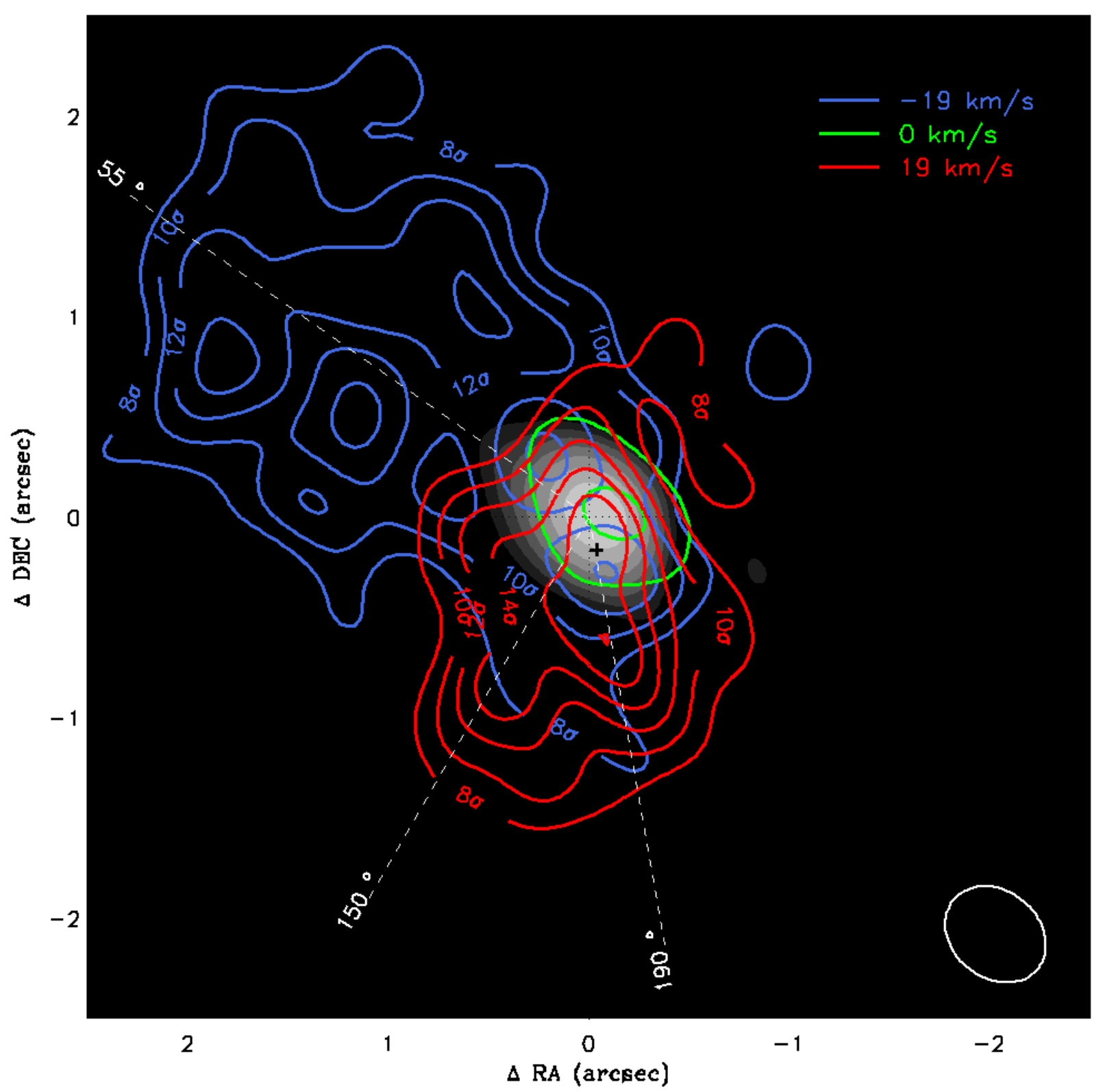

Fig. 13. - Map of the ${ }^{12} \mathrm{CO} J=2-1$ emission averaged over $3 \mathrm{~km} \mathrm{~s}^{-1}$ width for velocities $v_{z}=$ $-19 \mathrm{~km} \mathrm{~s}^{-1}$ (blue contours) and $19 \mathrm{~km} \mathrm{~s}^{-1}$ (red), compared to the contour map for the systemic velocity (green) and the $226.2 \mathrm{GHz}$ continuum emission map (gray-scaled image). Three position angles are marked to represent the outflow directions. The cross symbol refers to the position of companion star (blue component of two optical point sources in Monnier et al. 2000), assuming the red point source is located at the continuum peak position. The contour levels are $8 \sigma, 10 \sigma$, $12 \sigma, 14 \sigma, 16 \sigma$, and $18 \sigma$ for the high-end velocity channels (blue and red), and are $60 \sigma$ and $80 \sigma$ for the systemic velocity (green). The continuum map is logarithmically scaled from $3 \sigma$ to $50 \sigma$. The synthesized beam size is denoted by a white solid ellipse at the bottom right. 


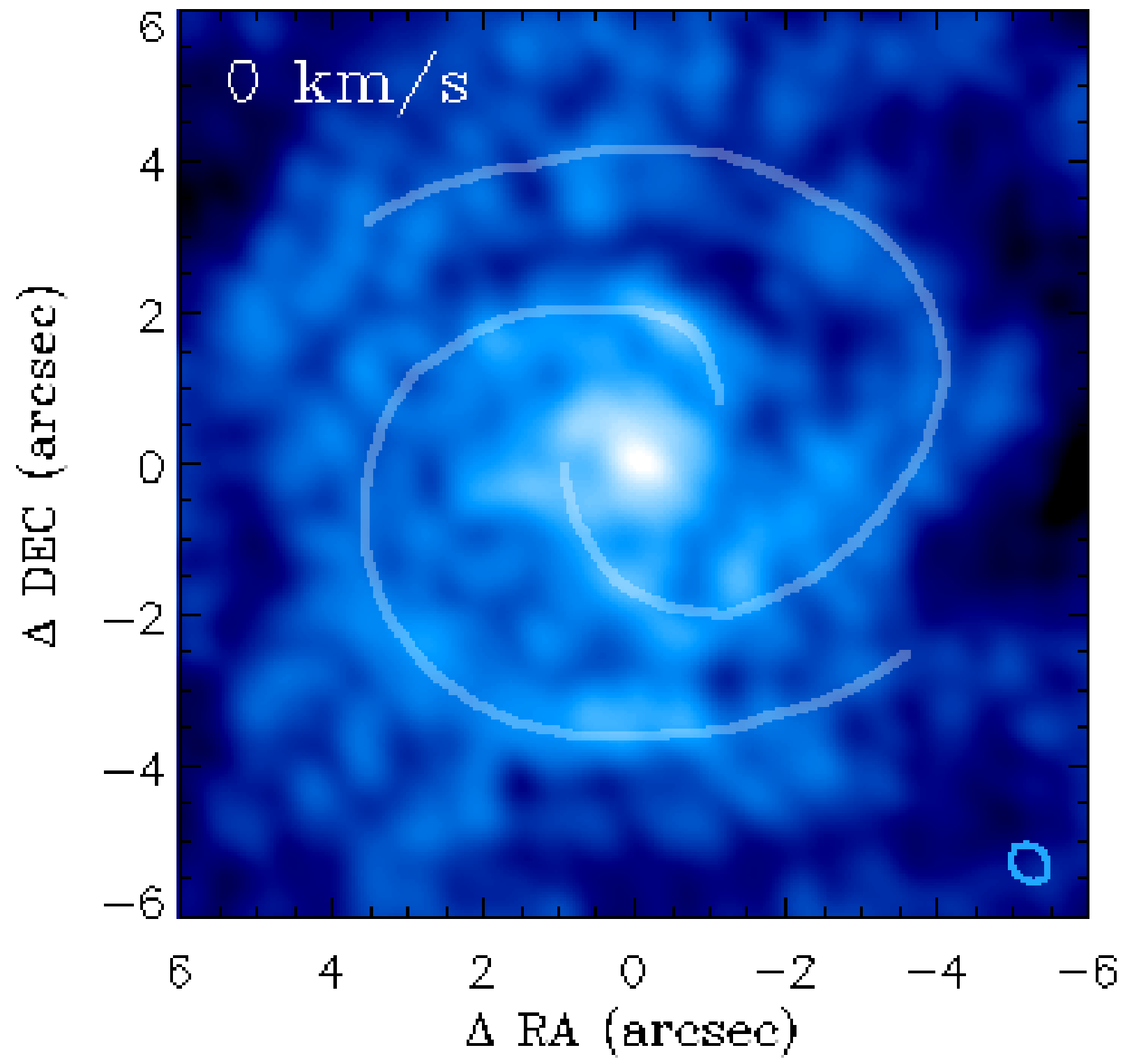

Fig. 14. - A double spiral feature appeared in the central region of ${ }^{12} \mathrm{CO} J=2-1$ map in the systemic velocity channel. Background image is the same as in the middle panel of Figure8, except for the smaller image size. 